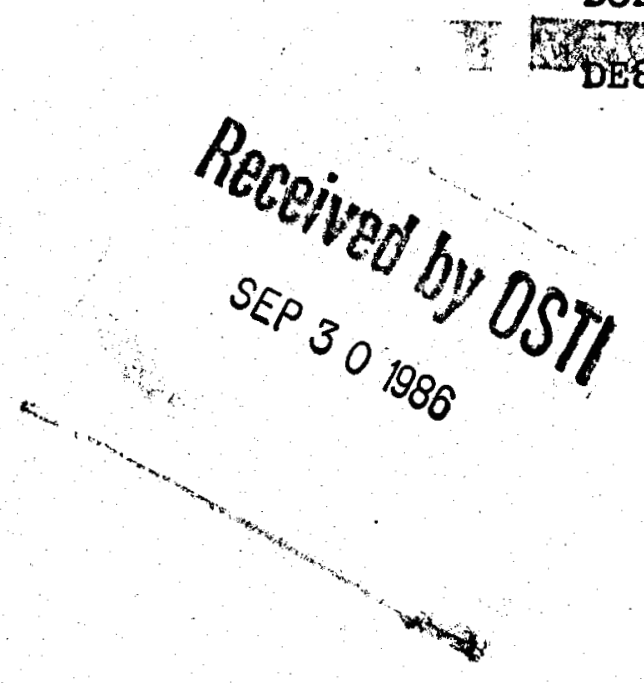

FINAL TECHNICAL REPORT

DE-AS03-83ER13100

\title{
ADVECTIVE-DIFFUSION/DISPERSION TRANSPORT OF CHEMICALLY REACTING SPECIES
}

Harold C. Helgeson, Profes\$or

Department of Geology and Gaophysics

University of California

Berkeley, Ca. 94609

Pefiod of Award: July 1, 1983 to June 30, 1985

Submitted June 20, 1986

\section{DISCLAIMER}

This report was prepared as an account of work sponsored by an agency of the United States Government. Neither the United States Government nor any agency thereof, nor any of their employees, makes any warranty, express or implied, or assumes any legal liability or responsibility for the accuracy, completeness, or usefuiness of any information, apparatus, product, or process disclosed, or represents that its use would not infringe privately owned rights. Reference herein to any specific commercial product, process, or service by trade name, trademark, manufacturer, or otherwise does not necessarily constitute or imply its endorsement, recommendation, or favoring by the United States Government or any agency thereof. The views and opinions of authors expressed herein do not recessarily state or reflect those of the United States Government or any agency thereof. 


\section{DISCLAIMER}

This report was prepared as an account of work sponsored by an agency of the United States Government. Neither the United States Government nor any agency Thereof, nor any of their employees, makes any warranty, express or implied, or assumes any legal liability or responsibility for the accuracy, completeness, or usefulness of any information, apparatus, product, or process disclosed, or represents that its use would not infringe privately owned rights. Reference herein to any specific commercial product, process, or service by trade name, trademark, manufacturer, or otherwise does not necessarily constitute or imply its endorsement, recommendation, or favoring by the United States Government or any agency thereof. The views and opinions of authors expressed herein do not necessarily state or reflect those of the United States Government or any agency thereof. 


\section{DISCLAIMER}

Portions of this document may be illegible in electronic image products. Images are produced from the best available original document. 


\section{PUBLICATION RESULTING FROM CONTRACT}

P.C. Lichtner, "Continuum model for simultaneous chemical reactions and mass transport in hydrothermal systems," Geochimica et Cosmochimica Acta, vol. $49,779-800,1985$ 
ADVECTIVE-DIFFUSIVE/DISPERSIVE TRANSPORT OF CHEMICALLY REACTING SPECIES IN HYDROTHERMAL SYSTEMS: FINAL REPORT FY83-85

\author{
P. C. Lichtner and H. C. Helgeson \\ Department of Geology and Geophysics \\ University of California \\ Berkeley, California 94720
}

\begin{abstract}
During FY83-85 a general formulation of multi-phase fluid flow coupled to chemical reactions was developed based on a continuum description of porous media. A preliminary version of the computer code MCCTM was constructed which implemented the general equations for a single phase fluid. The computer code MCCTM incorporates mass transport by advectiondiffusion/dispersion in a one-dimensional porous medium coupled to reversible and irreversible, homogeneous and heterogeneous chemical reactions. These reactions include aqueous complexing, oxidation/reduction reactions, ion exchange, and hydrolysis reactions of stoichiometric minerals. The code MCCTM uses a fully implicit finite difference algorithm. The code was tested against analytical calculations. Applications of the code included investigation of the propagation of sharp chemical reaction fronts, metasomatic alteration of microcline at elevated temperatures and pressures, and ion-exchange in a porous column. Finally numerical calculations describing fluid flow in crystalline rock in the presence of a temperature gradient were compared with experimental results for quartzite.
\end{abstract}




\section{INTRODUCTION}

Water-rock interactions play an important role in many geochemical processes of both practical and general geologic interest including chemical weathering, hydrothermal ore deposition, diagenesis, migration of toxic waste in groundwater, secondary mineral recovery processes, and containment of nuclear waste in an underground storage facility. A comprehensive mass transport model incorporating fluid flow and chemical reactions offers the possibility to better understand, and thereby to control and predict the time evolution of such processes. A quantitative model describing transport of fluids coupled to water-rock interactions can be very complex. Indeed the description of fluid flow in porous media in the absence of chemical reactions still contains many challenging problems, among them dispersivity in natural systems. Likewise the description of mass transfer involving heterogeneous reactions in the absence of fluid flow is extremely complicated. For a review see Evans (1979).

A description of reversible water-rock reactions between minerals and hydrothermal fluids coupled to fluid flow is complicated by the presence of sharp, propagating reaction fronts delineating different mineral assemblages. At such fronts, jump discontinuities may occur in the concentration, flux and reaction progress variables. Such problems are generally referred to as Stefan or moving boundary problems (Rubinstein,1971).

In recent work combining water-rock interactions with fluid flow, Norton and Taylor (1979) considered isotopic exchange problems, Nguyen et al. (1982), Nguyen et al. (1983) and Lasaga (1984) emphasized kinetic or irreversible aspects, while Walsh et al. (1984) considered a local equilibrium or reversible description: During FY83-85 Lichtner (1985a) developed a general theory of fluid flow in porous media coupled to water-rock interaction based on a continuum description incorporating both reversible and irreversible chemical reactions. This formulation incorporates reversible reactions as a limiting approximation of a presumably exact formulation of irreversible reactions within the continuum hypothesis. By eliminating the reaction rates corresponding to reversible reactions, a set of algebraic/partial 
differential equations were obtained for a minimal set of components referred to as primary species. These equations form the basis for the computer code MCCTM. They are solved using a fully implicit, finite difference algorithm for a single spatial dimension.

One unexpected result that emerged from time dependent calculations involving the metasomatic alteration of feldspar for conditions of steady, non-dispersive fluid flow, was the rapid (on a geologic time scale) formation of a steady state regime in which the spatial distribution of secondary minerals became stationary in time. This observation is expected to have significant consequences on future developments of coupling fluid flow to chemical reactions.

\section{WORK ACCOMPLISHED IN FY83-85}

During FY83-85 the following goals were accomplished:

- A general theory of fluid flow in porous media coupled to water-rock interaction based on a continuum representation of porous media was formulated and published (Lichtner, 1985a).

- A computer algorithm describing reversible precipitation and dissolution of solids coupled to mass transport by advection-diffusion/dispersion using an implicit finite difference method was developed and tested against exact results involving both transient and steadystate behavior (Lichtner et al., 1985a; Lichtner et al., 1985b).

- Development began on a preliminary version of the computer code MCCTM (MultiComponent Chemical Transport Model) which in its final form will account for mass and energy transport based on a Darcian description of multiphase fluid flow in porous media coupled to homogeneous and heterogeneous, reversible and irreversible chemical reactions. The present version of the code incorporates water-rock interaction coupled to the advectiondiffusion/dispersion equation employing a constant fluid velocity in a single spatial dimension. The chemical reactions provided for in the computer code MCCTM consist of reversible homogeneous reactions including ion pairing, complexing and redox reactions; and reversible and irreversible heterogeneous reactions consisting of hydrolysis reactions of minerals as 
well as reversible ion exchange reactions with a solid substrate. The rates of irreversible reaction of silicate minerals are described by the kinetic rate law developed by Aagaard and Helgeson (1982) and Helgeson et al. (1984) based on transition state theory.

- The computer code MCCTM was applied to the metasomatic alteration of K-feldspar (Lichtner et al., 1985c). Results of numerical calculations indicated that following a brief transient period, the continuum model yields a steady state flow regime which reproduces the results of a reaction path calculation for a closed system. (Actually the system is closed only with respect to the fluid phase, but open with respect to minerals.)

- A computer model describing fluid flow through crystalline rock in the presence of a temperature and pressure gradient coupled to precipitation/dissolution of quartz and incorporating changes in permeability and porosity was developed and compared with experiment (Morrow et al., 1985). Excellent agreement was obtained with experimental results over a short time behavior for flow through a quartzite rock in the presence of a temperature gradient (Lichtner et al., 1983).

\section{General Formulation of a Continuum Mass Transport Model}

\section{Continuum Mass Transport Equations}

Chemical reactions taking place in a system consisting of $N$ species denoted by $\mathbf{A}_{i}$, can be described by the general set of reactions:

$$
0=\sum_{i=1}^{N} \nu_{i r} \mathrm{~A}_{i},
$$

where the matrix $\nu_{i r}$ denotes the stoichiometric reaction coefficients taken to be positive for product species and negative for reactants. The reactions represented above consist of homogeneous reactions which take place within a single phase, and heterogeneous reactions which involve reactions between two or more phases. These include aqueous complexing, oxidation-reduction reactions, precipitation and dissolution of minerals and adsorption or ion exchange. Partial differential equations describing conservation of mass of the $i$ th species with provision for reversible and irreversible chemical reactions and fluid flow follow from 
the continuity equation modified to include a source/sink term consisting of the sum of the rates of the various reactions multiplied by the appropriate stoichiometric coefficients, according to

$$
\frac{\partial}{\partial t}\left(\phi_{\pi_{i}} C_{i}\right)+\nabla \cdot \mathrm{J}_{i}=\sum_{r, \text { reversible }} \nu_{i r} \frac{\partial \Xi_{r}}{\partial t}+\sum_{r, \text { irrev }} \nu_{i r} \frac{\partial E_{r}}{\partial t}(i=1, \ldots, N)
$$

where $C_{i}$ denotes the concentration of the $i$ th species, expressed in units of moles per unit volume of phase $\pi_{i}$ with volume fraction $\phi_{\pi_{i}}$ to which the species belongs, $J_{i}$ denotes the

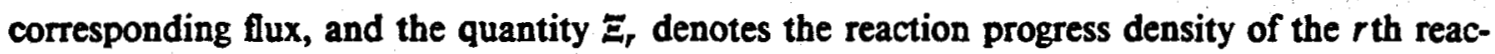
tion, expressed in units of moles per unit volume of bulk porous medium. The reaction progress density $\Xi_{r}$ is completely analogous to the usual reaction progress variable $\xi_{r}$ generally used to describe chemical reactions in closed or open systems which do not involve a space coordinate. The time derivative of the reaction progress density $\Xi_{r}$ is equal to the rate of the $r$ th reaction. The flux $J_{i}$ is given by the expression

$$
\mathrm{J}_{i}=-\phi_{\boldsymbol{x}_{i}} D_{i} \nabla C_{i}+v_{\boldsymbol{x}_{i}} C_{i}
$$

where $D_{i}$ denotes the diffusion coefficient of the $i$ th species and $v_{x_{i}}$ denotes the Darcy velocity of fluid phase $\pi_{i}$. The flux vanishes for mineral species.

The mass transport equations given in Eqn.(2) apply to both aqueous species and minerals. If the $i$ th species is contained in the aqueous phase of a saturated porous medium, then $\phi_{\pi_{i}}$ refers to the porosity $\phi$ of the porous medium. If, on the other hand, the $i$ th species denotes a stoichiometric mineral or the component of a solid solution, then $\phi_{\boldsymbol{\pi}_{i}}$ refers to the volume fraction occupied by the mineral and $C_{i}$ is equal to the reciprocal of the molar volume multiplied by the mole fraction $X_{i}$ of the $i$ th thermodynamic component describing the mineral:

$$
C_{i}=\bar{V}_{\pi_{i}}^{-1} X_{i}
$$

The volume fractions occupied by the various phases in the system must obey the volume conservation equation, which for a saturated porous medium with porosity $\phi$ has the form:

$$
\phi+\sum_{\pi=\text { mineral }} \phi_{\pi}=\phi_{R}
$$


where $\phi_{R}$ denotes the total reactive volume fraction of the system, including the aqueous phase.

The source/sink term represented by the right hand side of Eqn.(2) specifies the rate at which the $i$ th species is either created or destroyed as a result of chemical reactions. It is expressed as a sum of two terms, the first representing the contribution from reversible reactions and the second involving irreversible reactions. The reaction rates for reversible reactions must be determined by solving the transport equations augmented by the corresponding mass action equations given by

$$
K_{r}=\prod_{i=1}^{N} a_{i}{ }^{\prime i r},
$$

stipulating local equilibrium, where $K_{r}$ denotes the equilibrium constant for the $r$ th reaction and $a_{i}$ denotes the activity of the $i$ th species. For irreversible reactions constitutive relations must be supplied which express the reaction rate as a function of the composition, reacting surface area, temperature and pressure of the system (Aagaard and Helgeson, 1982; Helgeson et al., 1984).

\section{Generalized Rankine-Hugoniot Relations}

As a consequence of heterogeneous reactions taking place between minerals and the aqueous solution, sharp reaction fronts may exist delineating various mineral assemblages. Across such fronts, jump discontinuities in the concentration, flux and reaction progress density may occur. Conservation of mass at a reaction front requires that the following generalized Rankine-Hugoniot equations relating the velocity $v_{n}$ normal to the surface of discontinuity, denoted by $\Sigma$, to jump discontinuities in the field variables be satisfied;

$$
\mathbf{v}_{n}=-\frac{\partial \Sigma / \partial t}{\mathbf{n} \cdot \nabla \Sigma}=\frac{\left[\mathbf{J}_{i}\right] \cdot \mathbf{n}}{\left[\phi_{\pi_{i}} C_{i}\right]-\sum_{r} \nu_{i r}\left[\Xi_{r}\right]},
$$

where the square brackets $[\cdots]$ denote the jump in the enclosed quantity $(\cdots)$ across the surface $\Sigma$. It is important to note that the Rankine-Hugoniot equations are not an added assumption but can be shown to follow directly from the mass conservation equations them- 
selves provided the field variables appearing in the governing transport equations are interpreted as distributions (Lighthill, 1958) rather than ordinary functions (Lichtner, 1985a). In the case of transport by diffusion alone, the concentration is piecewise continuous with a jump discontinuity in slope at the reaction front. Advective transport results in a jump discontinuity in the concentration referred to as a chemical shock front (Rhee et al., 1970; Delhaye, 1974; Walsh et al., 1984).

\section{Mass Transport Equations for Primary Species}

The mass transport equations given by Eqn.(2) can be greatly simplified by eliminating the reaction rates corresponding to reversible reactions. This results in a reduced set of transport equations for a set of $N_{0}$ primary species, equal in number to the total number of species minus the number of reversible reactions, given by:

$\frac{\partial}{\partial t}\left\{\phi_{\pi_{j}} \Psi_{j}+\sum_{\substack{i=N_{0+1} \\ \pi_{i} \neq \pi_{j}}}^{N} \tilde{\nu}_{j i} \phi_{\pi_{j}} \bar{V}_{\pi_{i}}^{-1} X_{i}+\sum_{\alpha=1}^{N_{0}} \Phi_{j}^{\alpha}\right)+\nabla \cdot \Omega_{j}=\sum_{r=M_{0}+1}^{M} v_{j r} \frac{\partial \Xi_{r}}{\partial t}\left(j=1, \cdots, N_{0}\right)$,

where the generalized concentration field $\Psi_{j}$ and flux field $\mathbf{Q}_{j}$ are defined respectively by:

$$
\Psi_{j}=C_{j}+\sum_{\substack{i=N_{0}+1 \\ \pi_{j}=\pi_{j}}}^{N} \tilde{y}_{j i} C_{i}
$$

and

$$
\boldsymbol{Q}_{j}=\mathbf{J}_{j}+\sum_{i=N_{0}+1}^{N} \tilde{\nu}_{j i} \mathbf{J}_{i},
$$

the generalized adsorption isotherm $\Phi_{j}^{a}$ for exchange site $\alpha$ is given by

$$
\Phi_{j}^{\alpha}=C_{j}^{\alpha}+\sum_{i=N_{0}+1}^{N} \tilde{v}_{j i} C_{i}^{\alpha},
$$

where $C_{i}^{\alpha}$ denotes the solid phase concentration of the $i$ th species, and the matrices $\tilde{v}_{j i}$ and $\nu_{j r}^{\prime}$ are defined respectively by

$$
\tilde{\nu}_{j i}=-\sum_{r=1}^{M_{0}} \nu_{j r} \nu^{-1}{ }_{r i}
$$


and

$$
\nu_{j r}^{\prime}=\nu_{j r}+\sum_{i=N_{0}+1}^{N} \tilde{\nu}_{j i} \nu_{i r},
$$

where $j=1, \ldots, N_{0}$ refers to primary species, and $i=N_{0}+1, \ldots, N$ refers to secondary species. The matrix $\nu_{n i}^{-1}$ denotes the inverse to the square submatrix $y_{i r}$ where $i$ denotes a secondary species and $r$ refers to a reversible reaction.

The chemical reactions, excluding ion exchange reactions, have been arranged so that the first $M_{0}$ reactions represented by Eqn.(5), are reversible and can be written in the form

$$
\sum_{j=1}^{N_{0}} \tilde{v}_{j i} A_{j} \neq A_{i} \quad\left(i=N_{0}+1, \cdots, N\right),
$$

in which the primary species occur as reactants and the secondary species as products. Chemical reactions written in this form in which each reaction is associated with a single secondary species are referred to as being in canonical form. In general $\tilde{\nu}_{j i}$ can take on both positive and negative non-integral values. When $\tilde{\nu}_{j i}$ is non-negative, the generalized concentration field $\Psi_{j}$ is equal to the total concentration of the $j$ th primary species in phase $\pi_{j}$.

The remaining $M-M_{0}$ irreversible reactions can be formally expressed in terms of primary species alone according to

$$
0=\sum_{j=1}^{N_{0}} \nu_{j r} A_{j} \quad\left(r=M_{0}+1, \ldots, M\right)
$$

Ion exchange reactions may involve both primary and secondary species and are expressed in the form

$$
z_{j} A_{k}+z_{k} A_{j}\left(E_{\alpha}\right)_{z_{j}}=z_{j} A_{k}\left(E_{\alpha}\right)_{z_{k}}+z_{k} A_{j} \quad\left(\alpha=1, \ldots, N_{\sigma}\right)
$$

where $z_{k}$ denotes the valence of the $k$ th species, the species $\mathrm{E}_{\alpha}$ denotes a solid substrate exchange site of unit valence and $N_{\sigma}$ denotes the total number of exchange sites.

\section{Numerical Implementation}

The transport equations for the primary species given by Eqn.(8) combined with appropriate mass action constraints corresponding to reversible reactions provide the basis for 
the computer code MCCTM. They present a formidable set of coupled, nonlinear, algebraic/partial differential equations, which, in all but the simplest of cases, must be solved numerically. During FY83-85 a numerical algorithm was constructed employing an implicit finite difference method to solve the mass transport equations for the simplest case of an isothermal system in which fluid flow takes place with a constant Darcy velocity $v$ and diffusion/dispersion coefficient $D$ in a single spatial dimension. In this case the generalized flux $\Omega_{j}$ appearing in Eqn.(8) can be expressed in terms of the generalized concentration $\Psi_{j}$ according to:

$$
\mathbf{\Omega}_{j}=-\phi D \frac{\partial \Psi_{j}}{\partial x}+\nu \Psi_{j}
$$

The partial differential equations for the primary species are approximated by a set of ordinary differential equations in time by discretizing the spatial coordinate through subdividing the volume containing the system is into a finite set of $N_{D}$ open subsystems. Each subsystem corresponds to a representative elemental volume or control volume. The partial differential equations describing the system are averaged over each subsystem. The unknown variables, $N_{D} \times N_{0}$ in number, consist of the amplitudes of the concentration, volume fraction, and reaction progress density fields evaluated at each nodal point, taken to coincide with the center of each control volume. In the absence of fluid flow, these equations when applied to a single subsystem, reduce to equations familiar from the reaction path formulation for closed and open systems (Helgeson, 1968; Wolery, 1979; Reed, 1982; Helgeson and Murphy, 1983). The ordinary differential equations are integrated in time using a standard implicit finite difference technique in which they are approximated by a set of algebraic equations which provide values for the field variables at time $t+\Delta t$ in terms of previously obtained values at time $t$. Details may be found in Lichtner (1985a).

\section{Computation of Reaction Fronts}

By discretizing the mass transport equations on a fixed grid of node points with equal separation $\Delta x$, the added complications produced by the existence of sharp reaction fronts across which occur discontinuities in the field variables are ignored. It can be rigorously 
shown in certain special circumstances that such a simple discretization procedure yields a unique solution which converges to the exact result as the grid spacing goes to zero (Elliott and Ockendon, 1982). No attempt is made to determine the precise location of the boundaries separating different reaction zones, which remain uncertain to within $\Delta x$. However if the length of any given zone is large compared to the grid spacing, this inherent uncertainty is small. For this method, referred to as the weak formulation of the moving boundary problem (or enthalpy method), to be applicable requires formulating transport equations in such a manner that they incorporate the proper boundary conditions at the discontinuous reaction fronts. For example, by formulating energy conservation equations in terms of the enthalpy, the correct jump conditions are directly incorporated into the energy transport equations at a discontinuous phase change boundary as, for example, occurs in a solidification front; whence the name enthalpy method. The method has been successfully used to model freezing and melting problems in two-phase systems (Voller and Cross, 1981, 1983).

To implement this method a switching procedure is required to alter the set of independent variables as the configuration of phases change with distance and time. After a new composition is obtained at the end of a particular time step, it is necessary to ascertain whether any new phases have become supersaturated or existing phases have dissolved. If so, then appropriate steps must be taken to incorporate new heterogeneous constraint conditions at those nodal positions.

\section{Computer Code MCCTM}

The computer code MCCTM is presently applicable to a general system of $N$ chemically reacting species involving both reversible and irreversible reactions, undergoing transport by advection, diffusion and dispersion in a single spatial dimension coupled to water-rock interactions. The chemical algorithms presently included in the code are comparable to those found in the computer package EQ3/6 (Wolery, 1979) with the addition of ion exchange reactions. A thorough description for the theoretical basis of the model can be found in Lichtner (1985a). 
The code solves the partial differential equations given by Eqn.(8) describing mass transport in porous media subject to the mass action constraints corresponding to reversible homogeneous and heterogeneous reactions using an implicit finite difference algorithm. Input parameters include specification of the initial concentration of the primary species and mineral volume fractions at $t=0$ as a function of distance, boundary conditions specifying the concentration and/or flux of the primary species at the inlet and outlet of the porous column, Darcy flow velocity $v$, diffusion coefficient $D$, dispersion length $\alpha$, porosity $\phi$ of the porous medium, mineral molar volumes, and chemical data for the reactions included in the computation. For homogeneous and heterogeneous reversible reactions, this consists of the equilibrium constants and the stoichiometric reaction matrix $\tilde{v}_{j i}$, (see Eqn.(14)), Specification of irreversible reactions of minerals, requires entering the rate constants, the effective stoichiometric reaction matrix $\nu_{j r}^{\prime}$ (see Eqn.(13)), and reacting surface area expressed per unit volume of bulk porous medium. Ion exchange reactions require specification of the corresponding selectivity coefficients and the exchange capacity of the porous medium.

Other input parameters involve switching variables for various options. These include choice of flux or concentration boundary conditions, an option to compute activity coefficients using the Debye-Huckel model, a mode switch to provide for a closed system simulation without fluid flow equivalent to a reaction path run as performed by EQ6, and options to change the grid spacing and time step during the computation. In addition various I/O options are available for restarting, printing and plotting results.

To perform a calculation requires specifying a time-step array providing the time steps to be used in the computation. Often as a result of varying conditions due the presence of different mineral assemblages for example, the size of the maximum allowable time step can vary considerably with time. Generally this cannot be determined in advance and several trial runs are necessary before convergence in obtained.

\section{Code Verification}


To verify that the computer code MCCTM was correctly programmed as well as verifying that the various algorithms are functioning properly and approximating solutions to the partial differential equations representing conservation of mass, results of the code were carefully tested against analytical solutions and existing codes that have been independently tested. These tests included the following:

- Test of the code in the absence of chemical reactions. The code was compared with analytical solutions to the advection-diffusion/dispersion equation.

- Test of the chemical algorithms used in the code in the absence of fluid flow. The code was compared with the results of EQ3/6 for path type calculations in a closed system in which a single node point was used in MCCTM and the diffusion coefficient and fluid velocity were set equal to zero. The code MCCTM solves the mass transfer equations using an implicit finite difference algorithm which is completely different from the technique used by EQ6. Excellent agreement was obtained.

- Comparison of the steady-state limit obtained from transient calculations of interdiffusional mass transport incorporating precipitation/dissolution reactions with exact results obtained by Helfferich and Katchalsky (1970). Comparisons were made of the steadystate positions of the reaction zone boundaries, and concentrations and reaction rates as functions of distance (Lichtner et al., 1985a). In addition the method used by Helfferich and Katchalsky was generalized to multiple mineralic zones and compared with the steady-state limit of the corresponding transient calculation.

- Comparison with an analytical transient solution to the reaction-diffusion equations. An analytical solution to the reaction-diffusion equation for transient behavior was discovered which enabled a unique test of the numerical results (Lichtner et al., 1985b).

- Test of the ion exchange algorithm with independent results published by Valocchi et al. (1981) for multi-valent cation exchange. 


\section{Reaction-Diffusion Equations: Comparison of Exact and Numerical Results}

For the special case of diffusion of two solute species, $A$ and $B$, coupled to a single heterogeneous reaction, $A+B=C_{(s)}$ where $C_{(s)}$ denotes a solid phase, an exact solution was found to exist (Lichtner et al., 1985b). For the initial and boundary conditions consisting, respectively, of a solid initially in equilibrium with an aqueous solution and an aqueous solution diffusing into one end of a porous column of uniform cross sectional area which was not in equilibrium with the solid, the solid progressively dissolved forming a moving dissolution front propagating at a retarded velocity. Change in porosity resulting from dissolution of the solid was neglected. This example provided a unique test of predictions of an implicit finite difference algorithm based on a fixed grid of node points for the transient case.

A comparison between the exact result and results of the computer code MCCTM for the concentration of solute species $A$ and $B$ as a function of distance at time $t=1 \times 10^{5} \mathrm{sec}$ is shown in Fig. 1. A grid spacing of $\Delta x=0.02 \mathrm{~cm}$, time step of $\Delta t=10^{3} \mathrm{sec}$, a diffusion coefficient of $D=10^{-6} \mathrm{~cm}^{2} / \mathrm{sec}$ and porosity $\phi=0.2$ were used in the calculations. The initial volume fraction of the solid $\mathrm{C}_{(s)}$ was 0.1 , and the initial concentration of the solute species were $C_{\mathrm{A}}=C_{\mathrm{B}}=1$ mole/liter. The injected solution had a concentration of $C_{\mathrm{A}}=0.5$ and $C_{\mathrm{B}}$ $=0$ moles/liter. The numerical results are represented by the circles and the exact solution by the solid lines. As can be seen, the agreement at the particular time is excellent. The kinks in the concentration occurring at approximately $0.2 \mathrm{~cm}$ from the inlet represent a sharp reaction front where the solid $C_{(s)}$ is dissolving. The gradient of each solute species has a jump discontinuity across the dissolution front. To the left of the position of the kinks complete dissolution of the solid has occurred. To the right of the kink precipitation occurs, the rate decreasing with distance from the front.

More insight into the differences between the numerical calculation and the exact result can be obtained by comparing the evolution of the position of the dissolution front with time as shown in Fig. 2. Here one notes a striking difference between the two approaches. While the exact solution yields a smooth curve given by the expression 


$$
\begin{gathered}
-14, \\
x_{0}(t)=2 \gamma \sqrt{D t},
\end{gathered}
$$

where the coefficient $\gamma$ is a function of the initial and boundary conditions only and is independent of the diffusion coefficient $D$, the implicit finite difference calculation yields a step-function like curve with step height given by the spacing between node points. However, as is apparent from the figure, the two results agree exactly at certain instants in time. For earlier or later times however, the numerical result overshoots or undershoots respectively the exact position of the dissolution front. This type of behavior is typical of the weak formulation of the moving boundary problem (Voller et al., 1979; Voller and Cross, 1981, 1983).

\section{Counter Diffusion and the Steady-State Limit}

A further comparison between exact and numerical calculations was provided by counter-diffusion of two solute species A and B reacting reversibly to form various solid precipitates (Lichtner et al., 1985a). Excellent agreement was obtained between results of the steady-state limit of numerical solutions for the transient case and the analytical steady-state solution obtained by Helfferich and Katchalsky (1970) for a single solid phase. Their results were also generalized to the case of two solid phases. Comparisons were made for the reaction zone boundaries, concentration profiles and reaction rates.

The next two examples considered involve a single solid phase $C_{(s)}$ reacting with the fluid according to $A+B \neq C_{(s)}$ with the geometry shown in Fig. 3. The transient behavior of the zone boundary is illustrated in Fig. 4 where the position of the reaction front is plotted as a function of time for the case of symmetrical boundary conditions $\hat{C}_{A}^{0}=\hat{C}_{B}^{\prime}=1$, equal diffusion coefficients $D_{A}=D_{B}=D$ with $D=1 \times 10^{-6} \mathrm{~cm}^{2} / \mathrm{sec}$, and equilibrium constant $\hat{K}=1 \times 10^{-6}$. An arbitrary value of one was used for $\hat{V}_{s}$. As noted by Helfferich and Katchalsky (1970), equal diffusion coefficients result in precipitation beginning at the center of the column in the plane perpendicular to its axis. The reaction zone grows symmetrically with time about the center of the column as a result of the symmetrical boundary conditions, and thus only the left hand boundary is plotted in Fig. 4. Precipitation begins at approximately $1.2 \times 10^{4} \mathrm{sec}$. The width of the reaction zone rapidly increases with time passing through 
point $a$ at $t_{a}=1.3 \times 10^{4} \mathrm{sec}$ corresponding to a width of approximately $0.32 \mathrm{~cm}$, until it reaches a maximum value of approximately $0.66 \mathrm{~cm}$ at $t_{b}=2 \times 10^{4} \mathrm{sec}$ labeled by the point $b$. The outer edges of the reaction zone then dissolve, its width decreasing with time until steady-state is reached at $t_{c}=1.6 \times 10^{6} \mathrm{sec}$ labeled by the point $c$. The width of the reaction zone at steady-state is approximately $0.5 \mathrm{~cm}$, in excellent agreement with the exact steadystate value obtained from the analytical calculation. Contrary to statements made by Helfferich and Katchalsky (1970), however, the time required to reach steady-state is much longer than they suggest. This is a result of reaction with the solid $C_{(s)}$. The slow dissolution at the edges of the reaction zone prolongs the time required to reach steady-state. Again a discontinuous curve describing the position of the reaction front is obtained. This curve presumably overshoots and undershoots the exact position of the reaction front.

Next asymmetrical boundary conditions with $\hat{C}_{A}^{0}=2$ and $\hat{C}_{B}^{l}=0.5$ are considered. The equilibrium constant has the value $\hat{K}=10^{-2}$. The same values for the diffusion coefficients are used as in the previous example. The positions of the reaction fronts versus time are plotted in Fig. 5. Precipitation begins at the center of the column at approximately $5 \times 10^{4} \mathrm{sec}$. As the reaction zone grows, it shifts position moving to the right as a result of the asymmetric boundary conditions. In this case dissolution occurs only at the left zone boundary. Steady state is reached after approximately $5 \times 10^{6} \mathrm{sec}$. Dimensionless concentration profiles are plotted in Fig. 6 versus distance for times $6 \times 10^{4} \mathrm{sec}$ and $4.3 \times 10^{6} \mathrm{sec}$, the latter time corresponding to steady-state. Although not apparent from this figure, for times earlier than steady-state, there exist kinks in the concentration profiles at the reaction zone boundaries. As steady-state is approached, the kinks disappear in agreement with the Rankine-Hugoniot relations (Eqn.(7)), since at steady-state the zone boundaries become stationary. The precipitation reaction rate is plotted in Fig. 7 for the asymmetric case.

Finally two solid phases $C_{1(s)}$ and $C_{2(s)}$ are considered reacting according to

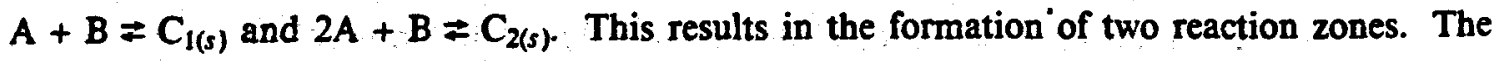
equilibrium constants are taken to have the values $\hat{K}_{1}=10^{-4}$ and $\hat{K}_{2}=10^{-5}$. Symmetrical 
boundary concentrations are considered with the values $\hat{C}_{A}^{0}=\hat{C}_{B}^{\prime}=1$, and equal diffusion coefficients are used with values $D_{A}=D_{B}=10^{-6} \mathrm{~cm}^{2} / \mathrm{sec}$. The positions of the reaction zone boundaries are plotted as a function of time in Fig. 8. Precipitation begins at slightly different times for each solid, occurring first for the solid $C_{1(s)}$. As the system evolves in time and the reaction zones grow, the inner reaction zone boundaries collide with one another forming a sequence of invariant points at which both solids coexist. At an invariant point the concentrations of the aqueous species are completely determined by the mass action equations and thus must be independent of time. An invariant region of space, as opposed to a point, cannot exist since in such a case the mass action equations could not all be satisfied simultaneously by continuous (differentiable) functions of distance. According to Fig. 8, invariants points occur at times given approximately by $2.8 \times 10^{4}, 3.3 \times 10^{4}, 3.65 \times 10^{4}, 4.2 \times 10^{4}$, 4.9-5.05 $\times 10^{4}$, and $6.2-6.5 \times 10^{4} \mathrm{sec}$. These values, however, depend on the size of the grid spacing. In an exact solution the invariant point would undoubtedly move continuously, disappearing as steady-state is approached. Eventually the inner reaction fronts separate entirely and remain separated at steady-state in agreement with the exact steady-state limiting solution (Lichtner et al., 1985a).

\section{Chemical Shock Fronts}

Evidence for sharp reaction fronts in hydrothermal systems has been observed in experiments by Morey and Fournier (1961) involving the dissolution of Nepheline at $295{ }^{\circ} \mathrm{C}$. An example involving chemical shock fronts in which jump discontinuities in concentration and flux occur at reaction zone boundaries is shown in Fig.9. This example is similar to that presented by Walsh et al. (1984), and involves four aqueous species with concentrations denoted by $C_{1}, C_{2}, C_{3}$, and $C_{4}$ which react to form three solid phases, $M_{12}, M_{13}$, and $M_{24}$ according to the mass action equations given by

$$
K_{i j} C_{i} C_{j}=1 \text {, }
$$

with $K_{12}=1, K_{13}=2$ and $K_{24}=0.5$. Transport occurs by advection and diffusion with a Darcy flow rate of $1 \mathrm{~m} / \mathrm{yr}$, and a diffusion coefficient of $10^{-6} \mathrm{~cm}^{2} / \mathrm{sec}$. Jump discontinuities in the 
concentration, flux and volume fraction occur across each reaction front indicated by the three dashed vertical lines. The initial aqueous solution consists of species 1 and 2 with concentrations $C_{1}=C_{2}=1$ in equilibrium with solid $M_{12}$ with an initial volume fraction of 0.1 . An aqueous solution with concentrations $C_{1}=0.5, C_{2}=0, C_{3}=1.5$ and $C_{4}=2$, is injected at one end of the column. Note that although the curves as drawn in Fig. 9 appear to be piecewise continuous because of the straight lines drawn between each of the 100 node points used in the calculation, they actually contain jump discontinuities at the reaction fronts as a result of the advective flow velocity. As noted by Walsh et al. (1984), downstream equilibrium conditions are approximately satisfied even though no solid phases are present.

The position of the shock fronts as a function of time are shown in Fig. 10. Four reaction zones occur. In the first zone no solids are present. The second zone consists of solid $M_{24}$, the third zone consists of solids $M_{13}$ and $M_{24}$ and the fourth zone consists of solid $M_{12}$. Note that each front moves with a different average retarded velocity. Again note the stepfunction like curves, characteristic of the weak formulation of the moving boundary problem. The volume fractions of solids $M_{13}$ and $M_{24}$ are plotted in Fig. 11 as a function of distance at $t=9 \times 10^{4} \mathrm{sec}$. Solid $M_{12}$ occurs to the right of the common boundary of solids $M_{13}$ and $M_{24}$.

\section{Metasomatic Alteration of $K$-feldspar}

As a preliminary example of water-rock interactions relevant to weathering processes, ore deposition and metasomatic alteration, the computer code MCCTM was used to describe percolation and diffusion of a dilute $\mathrm{HCl}$ solution through a porous medium initially consisting of K-spar in equilibrium with an aqueous solution at $100^{\circ} \mathrm{C}$. The composition of the initial fluid had a pH of $6, C_{\mathrm{K}^{+}}=10^{-3}$ moles/liter and was just undersaturated with respect to amorphous silica. Quartz was considered to be inert in the calculations. The porosity of the porous medium was 0.2 . The injected $\mathrm{HCl}$ solution had a $\mathrm{pH}$ of 5 . A constant Darcy flow velocity of $1 \mathrm{~m} / \mathrm{yr}$ and a diffusion coefficient of $10^{-6} \mathrm{~cm}^{2} / \mathrm{sec}$ were used in the calculations. The microcline reacted irreversibly according to the rate law proposed by Helgeson $e t$ al. (1984). The surface area per unit volume of bulk porous medium used in the calculations was 30 
$\mathrm{cm}^{-1}$

corresponding to $2 \mathrm{~mm}$ sized cubical grains. The chemical reactions included in the calculation are given in Table 1. The secondary mineral phases, gibbsite, kaolinite, muscovite and pyrophyllite, formed as a result of dissolution of the microcline, and were assumed to be in local equilibrium with the fluid. Homogeneous reactions included in the calculation consisted of the dissociation of $\mathrm{H}_{2} \mathrm{O}$, and the speciation of $\mathrm{Al}^{3+}$ and $\mathrm{SiO}_{2}$. These reactions were considered reversible, generally an excellent approximation for complexing reactions.

The evolution of the positions of the resulting reaction zone boundaries with time are shown in Fig. $12 \mathrm{a}$ for times up to $9 \times 10^{4} \mathrm{sec}$. As illustrated, there is a rather complicated behavior of phases appearing and disappearing until eventually only two reaction zones consisting of gibbsite and muscovite, survive. At $t \approx 2.5 \times 10^{4} \mathrm{sec}$ all four secondary minerals are present in the order: gibbsite-kaolinite-muscovite-pyrophyllite. Note that the various phase boundaries generally have different slopes, indicating different velocities and hence different retardation factors. In Fig. $12 \mathrm{~b}$ the zone boundaries for muscovite and gibbsite are plotted for times up to $5.5 \times 10^{5} \mathrm{sec}$. The gibbsite-muscovite boundary becomes essentially stationary at approximately $1.5 \mathrm{~cm}$ from the inlet, while the leading muscovite boundary moves with a constant, slightly retarded velocity.

The volume fractions corresponding to muscovite and gibbsite are plotted in Fig. 13 as a function of distance for the times 3,4 and $5 \times 10^{5} \mathrm{sec}$. The gibbsite-muscovite boundary is approximately stationary in time, with internal precipitation resulting in an increase in the volume fractions of both minerals with time.

The $\mathrm{pH}$ of the aqueous solution is plotted in Fig. 14 as a function of distance for various times. A steady-state limit is obtained through the gibbsite field and progressively extending into the muscovite field. A sharp increase in $\mathrm{pH}$ is obtained at the inlet where gibbsite is formed.

The sequence of secondary mineral phases formed at different times can be better under- 
stood by projecting the composition of the aqueous solution along the flow path onto an activity diagram (Fig. 15). Each curve represents the fluid composition as a function of distance, the left hand endpoint corresponding to the inlet and the common terminating point corresponding to the initial fluid composition in equilibrium with K-spar.

Also plotted on this diagram (dashed curve) is the reaction path for a closed system in which microcline dissolves irreversibly with the identical initial fluid composition as the injected fluid in the transport problem. The steady-state limit obtained along the flow path coincides with the closed system calculation as demonstrated in the figure.

The reaction rate of microcline is plotted in Fig. 16 for $t=5 \times 10^{5}$ sec. In addition to the dissolution region extending to approximately $6 \mathrm{~cm}$, there is a region of precipitation followed by a region of subsequent dissolution.

\section{Ion-Exchange}

The effect of aqueous complexing on retardation of solute species resulting from ion exchange reactions in groundwater can be important for certain geochemical systems (Wangen et al., 1982; Wangen and Stallings, 1984). Using the computer code MCCTM the effect of ion complexing was investigated for several hypothetical systems. Solid phases other than the adsorption substrate were not included in the calculations. Exchange took place between a mono-valent cation such as $\mathrm{Na}^{+}$and a cation of valence +3 denoted by $\mathrm{M}^{3+}$. The exchange capacity of the porous medium was fixed at a ratio of aqueous to solid concentration of 10 . An average pore velocity of $1 \mathrm{~m} / \mathrm{yr}$ and diffusion coefficient of $1 \times 10^{-5} \mathrm{~cm}^{2} / \mathrm{sec}$ were used in the calculations. The concentration profiles of the solute species after a time of 5 years have elapsed are shown in Fig. 17a in the absence of ion-complexing, for an initial aqueous solution of $0.01 M \mathrm{NaCl}$ and injected solution containing $0.001 M \mathrm{M}_{2} \mathrm{~L}_{3}$, where $L^{2-}$ denotes a hypothetical anion, in addition to the initial $\mathrm{NaCl}$ solution. The ion-exchange selectivity coefficient used in the calculation was equal to 1 . The advancing front of $\mathrm{Na}^{+}$and the anion $\mathrm{L}^{2-}$ move unretarded with the fluid velocity whereas the cation $\mathrm{M}^{3+}$ is seen to be retarded by a factor of approximately 10 . The corresponding ion-exchange reaction rate is shown in Fig. 
$17 \mathrm{~b}$.

In Figs. 18a,b is shown the solute concentration profiles that result when the complexes $\mathrm{NaL}^{-}$and $\mathrm{ML}^{+}$are included with corresponding equilibrium constants $K_{\mathrm{ML}^{+}}=10^{4.15}$ taken from the reaction $\mathrm{Fe}^{3+}+\mathrm{SO}_{4}^{2-} \rightleftarrows \mathrm{FeSO}_{4}$, and $K_{\mathrm{NaL}^{-}}=10^{0.7}$. Fig. 18a corresponds to the same conditions as in Figs. 17a,b. Fig. $18 \mathrm{~b}$ corresponds to a $0.01 M \mathrm{M}_{2} \mathrm{~L}_{3}$ solution in addition to a $0.01 \mathrm{M} \mathrm{NaCl}$ solution. Retardation is reduced as a result of the complexing and the increased concentration of the feed solution. The complexes did not to take part in the exchange reactions.

Figs. 19a,b demonstrate the effect of increased complexing on retardation. In Fig. 19a the equilibrium constant for the association reaction for $\mathrm{ML}^{+}$was taken as $10^{6}$ and in Fig. $19 \mathrm{~b}$ as $10^{8}$. Such values for the equilibrium constants of complexing reactions are not unusual for organic chelating agents. As expected, the larger the equilibrium constant for the association reaction, the greater the reduction in retardation.

The final example considered involves the injection of a $0.01 M$ solution of $\mathrm{H}_{2} \mathrm{SO}_{4}$ and $\mathrm{NaCl}$ containing $0.001 M \mathrm{M}_{2}\left(\mathrm{SO}_{4}\right)_{3}$ into a initial solution of $0.01 \mathrm{M} \mathrm{NaCl}$. The dissociation of water was included in the calculation. The result for the solute concentration profiles after 5 years is shown in Fig. 20. Fig. 21 contains the $\mathrm{H}^{+}$and $\mathrm{OH}^{-}$concentration profiles as well as the complexes $\mathrm{HSO}_{4}^{-}$and $\mathrm{MOH}^{++}$neither of which took part in ion-exchange reactions. The concentration of the cation $M^{3+}$ is approximately two orders of magnitude less than the concentration of the complex $\mathrm{MSO}_{4}^{-}$, for which the corresponding isopleths have moved approximately twice as far.

\section{Fluid Flow in Crystalline Rock: Changes in Permeability and Porosity}

It remains an open question whether mass transport equations based on a continuum description of fluid flow in a porous medium can be applied to crystalline rock where fluid flow presumably takes place along grain boundaries and through microcracks. Experiments by Morrow et al. (1981), Moore et al. (1982), Vaughan and Byerlee, (1984) and Morrow 
et al. (1985), offer a unique opportunity to test the continuum mass transport model for fluid flow through crystalline rock. Experiments were carried out with cylindrical samples of granite, quartzite, anorthosite and gabbro, $7.6 \mathrm{~cm}$ in diameter and $8.9 \mathrm{~cm}$ long containing a central borehole of radius $1.27 \mathrm{~cm}$. Hot fluid was injected into the borehole and flowed radially outward as a result of a pressure gradient imposed across the sample. The temperature within the sample rapidly reached its steady-state value with a radial profile of the form

$$
T(r)=\frac{T_{2}-T_{1}}{\ln \frac{r_{2}}{r_{1}}} \ln \frac{r}{\sqrt{r_{1} r_{2}}}+\frac{T_{2}+T_{1}}{2}
$$

where $r_{1}$ and $r_{2}$ denote the inner and outer radii of the cylinder respectively held at respective temperatures $T_{1}$ and $T_{2}$.

Several attempts have been made to describe these experiments with varying degrees of success (Lichtner et al., 1983; Keith et al., 1984). Numerical models employing a Darcy description of fluid flow with a power law dependency of permeability on porosity of the form

$$
\kappa=\kappa_{0}\left[\frac{\phi}{\phi_{0}}\right]^{n}
$$

with $n=2$ and 3 , where $\kappa_{0}$ and $\phi_{0}$ denote the initial permeability and porosity of the system were used to model the experiments. Partial differential equations representing mass conservation were solved numerically employing a radially symmetric spatial mesh with uniform spacing by modifying the computer code MULKOM (Pruess, 1983) to include both reversible and irreversible precipitation and dissolution of quartz. Kinetic rate parameters were taken from Rimstidt and Barnes (1980), and the solubility of quartz was computed using an efficient algorithm developed by Fournier and Potter (1982). The quartzite was assumed to be in equilibrium with the initial fluid at $111^{\circ} \mathrm{C}$ and the injected fluid was in equilibrium with quartz at $250^{\circ} \mathrm{C}$.

It was generally found that the early time behavior of the experiments could be reproduced but not the long term behavior. This is not surprising given the complicated nature of the rocks used in most of the experiments. Shown in Fig. 22 are the predictions resulting 
from calculations using the computer package EQ3/6 for the mineral volume changes obtained by cooling an aqueous solution in equilibrium with a hypothetical rock from $300{ }^{\circ} \mathrm{C}$ to $100{ }^{\circ} \mathrm{C}$ in a closed system. Results are compared for a rock of roughly the same composition as Barre granite used in some of the experiments, with a rock composed entirely of quartzite. The solid lines labeled quartz, muscovite, microcline and low albite refer to the hypothetical granite while the dot-dashed line refers to quartzite. The dashed line gives the total volume change of the granite rock as it is cooled. As can be seen, the large increase in quartz for the granite rock is compensated by the dissolution of low albite and microcline, a possible explanation for the inability to fit the experimental data with a model based on the reaction of quartz alone. Furthermore the volume increase for quartzite was larger than for the granite, consistent with the experimental results.

Somewhat better agreement with experiment was obtained for quartzite than was the case for granite. Preliminary results for the prediction of the reduction in flow rate with time is shown in Fig. 23. The solid curve refers to the irreversible precipitation of quartz for an initial porosity of $7 \times 10^{-4}$, initial permeability of $1.89 \times 10^{-19} \mathrm{~m}^{2}$ and surface area per unit volume of bulk porous medium of $7.7 \times 10^{6} \mathrm{~m}^{-1}$. An excellent fit to the experimental data (Morrow et al., 1985) was obtained for times up to approximately 100 hours during which the flow rate dropped by two orders of magnitude. For longer times the numerical calculations gave too great a reduction in flow rate compared to the experimental results which indicated a linear decrease with time (see insert Fig. 23). The dashed curve is the result of the local equilibrium approximation with the same initial porosity and permeability as used in the irreversible calculation. The substantial difference between the two results clearly indicates that the flow rate in this experiment was "fast" relative to the rate of precipitation of quartz which was found to be approximately independent of the flow velocity.

In Fig. 24 the predicted change in porosity as a function of distance is plotted for different times. According to the numerical calculations, maximum deposition of quartz occurs at approximately $12 \mathrm{~mm}$ from the axis of the cylinder or approximately $6 \mathrm{~mm}$ inside 
the sample. This is a result of two competing effects: (1) the reduction in reaction rate with temperature; and (2) the increase in rate resulting from increasing supersaturation of $\mathrm{H}_{4} \mathrm{SiO}_{4}$ with distance. The fluid in the bore hole and thus the fluid entering the sample was assumed to be in equilibrium with quartz at the inlet temperature. The degree of supersaturation is indicated in Fig. 25 where the mass fraction of $\mathrm{SiO}_{2(a q)}$ is plotted (solid lines) as a function of distance for different times. The dashed line is the equilibrium value for the steady-state temperature profile within the sample. A steady-state value is obtained after approximately 100 hours. In contrast to the result for irreversible reaction of quartz, the local equilibrium approximation results in a sharp reduction in porosity at the inlet as shown in Fig. 26.

At the point of maximum deposition of quartz there is a sharp drop in fluid pressure shown in Fig. 27 and a corresponding peak in the average pore velocity shown in Fig. 28.

The above results offer encouragement in applying the continuum description of fluid flow in porous media to crystalline rock where extremely small porosities $\left(\approx 10^{-3}\right)$ and permeabilities $\left(\approx 10^{-19} \mathrm{~m}^{2}\right)$ occur. Future calculations will concentrate on rock types such as granite, anorthosite and gabbro involving more complicated mineral assemblages.

\section{SUMMARY}

A general theory was formulated describing mass transport in porous media coupled to chemical reactions including fluid-rock interaction. An algorithm for coupling mass transport by advection, dispersion and diffusion with both homogeneous and heterogeneous chemical reactions has been successfully implemented into the computer code MCCTM. The code was tested by comparison with analytical solutions, with special regard to the use of a fixed set of node points to adequately describe moving chemical reaction fronts. It was found that usual finite difference procedures employing a discrete set of fixed node points are adequate to describe the position of reaction fronts provided the width of the overall reaction zone is large compared to the grid size. Two and three dimensional problems will undoubtedly require explicit front tracking methods. The model was applied to the metasomatic alteration of 
feldspar and ion-exchange in a porous column. One important result obtained from the calculations for the alteration of feldspar was the occurrence of a steady state regime in which mineral reactions zones become stationary in time, contrary to the expected behavior of continued propagation in response to fluid flow. Finally fluid flow in crystalline rock in the presence of a temperature gradient with provision for precipitation and dissolution of quartz was investigated. The results of numerical calculations for the reduction in the fluid flow rate with time compared favorably with experiment for early time behavior, but not for long times where the theoretical calculations predicted a more rapid reduction in the flow rate than observed. Work accomplished during FY83-85 has demonstrated the feasibility of developing a comprehensive quantitative description for the transport of fluids in the Earth's crust and mantle. 


\section{REFERENCES}

Aagaard, P. and Helgeson H. C. (1982) Thermodynamic and kinetic constraints on reaction rates among minerals and aqueous solutions, I. Theoretical considerations. Am. J. Sci. $282,237-285$.

Delhaye, J. M. (1974) Jump conditions and entropy sources in two-phase systems. Local instant formulation. Int. J. Multiphase Flow 1, 395-409.

Elliott, C. M. and Ockendon, J. R. (1982) Weak and variational methods for moving boundary problems, 213 pp. Pitman.

Evans, J. W. (1979) Mass transfer with chemical reaction. Minerals Sci. Engng. 11, 207-223.

Fournier R. O. and Potter II, R. W. (1982) An equation correlating the solubility of quartz in water from $25^{\circ} \mathrm{C}$ to $900^{\circ} \mathrm{C}$ at pressures up to 10,000 bars. Geochimica et Cosmochimica Acta 46, 1969-1973.

Helfferich, F. and Katchalsky, A. (1970) A simple model of interdiffusion with precipitation. J. Phys. Chem. 74, 308-314.

Helgeson, H. C. (1968) Evaluation of irreversible reactions in geochemical processes involving minerals and aqueous solutions-I. Thermodynamic relations. Geochim. Cosmochim. Acta 32, 853-877.

Helgeson, H. C. (1970) A chemical and thermodynamical model of ore deposition in hydrothermal systems. In: Mineralogical Society of America, Fiftieth Anniversary Symposium, Special Paper \#3, Ed. B. A. Morgan, 155-186.

Helgeson, H. C. and Murphy, W. M. (1983) Calculation of mass transfer among minerals and aqueous solutions as a function of time and surface area in geochemical processes. 1. Computational approach. Math. Geol. 15, 109-130.

Helgeson, H. C., Murphy, W. C. and Aagaard, P. (1984) Thermodynamic and kinetic constraints on reaction rates among minerals and aqueous solutions. II. Rate constants, effective surface area, and the hydrolysis of feldspar. Geochimica et Cosmochimica Acta 
48, 2405-2432.

Keith, L. A., Delaney, P. T. and Moore, D. E. (1984) Permeability Reduction due to Precipitation of Quartz under Nonisothermal Conditions, Proc. Ninth Workshop on Geothermal Reservoir Engineering, Stanford University, Stanford, California.

Lasaga, A. C. (1984) Chemical kinetics of water-rock interactions. J. Geo. Res. 89, 40094025 .

Lerman, A. (1979) Geochemical processes: water and sediment environments. John Wiley and Sons, $481 \mathrm{pp}$.

Lichtner, P. C. (1982) Transport of chemically reacting solutes in groundwater. Trans. American Geophysical Union (Abstract) 63, 929.

Lichtner P. C. (1985a) Continuum model for simultaneous chemical reactions and mass transport in hydrothermal systems. Geochimica et Cosmochimica Acta 49, 779-800.

Lichtner, P. C. and Helgeson, H. C. (1982) Chromatographic transport of aqueous species in hydrothermal systems. I. Calculation of concentration profiles and retarded velocities in solutions coexisting with alkali feldspars at magmatic temperatures and pressures. Geological Society of America, Abstracts with Programs 14, 546.

Lichtner, P. C. and Helgeson, H. C. (1984) Chemical shock fronts induced by advectivediffusive/dispersive flows in hydrothermal systems. Trans. American Geophysical Union (Abstract) 65, 1126.

Lichtner, P. C., Helgeson, H. C. and Pruess, K. (1983) Numerical modeling of fluid flow with simultaneous chemical reactions in hydrothermal systems. Symposium on fluid flow in crystalline rocks at elevated temperatures, Geological Society of America, Abstracts with Programs 15, 627.

Lichtner, P. C., Oelkers, E. H., and Helgeson, H. C. (1985a) Interdiffusion with multiple precipitation/dissolution reactions: Transient model and the steady-state limit. In preparation. 
Lichtner, P. C., Oelkers, E. H., and Helgeson, H. C. (1985b) Exact and numerical solutions to the moving boundary problem resulting from reversible heterogeneous reactions and aqueous diffusion in a proous medium. In preparation.

Lichtner, P. C., Murphy, W. M., and Helgeson, H. C. (1985c) Metasomatic alteration of feldspar. In preparation.

Lighthill, M. J. (1958) Introduction to Fourier Analysis and Generalized Functions, 79 pp. Cambridge University Press.

Morey, G. W. and Foumier, R. O. (1961) The decomposition of microcline, albite and nepheline in hot water. American Mineralogist 46, 688-699.

Morrow, C. A., Moore, D. E. and Byerlee, J. D. (1985) Permeability changes in crystalline rocks due to temperature: effects of mineral assemblage. Material Research Society Proceedings 27.

Nguyen, V. V., Gray, W. G., Pinder, G. F., Botha, J. F., and Crerar, D. A. (1982) A theoretical investigation on the transport of chemicals in reactive porous media. Water Resources Research 18, 1149-1156.

Nguyen, V. V., Pinder, G. F., Gray, W. G. and Botha, J. F. (1983) Numerical simulation of uranium in -situ mining. Chem. Eng. Sci. 38, 1855-1862.

Norton, D. and Taylor, H. P. Jr. (1979) Quantitative simulation of the hydrothermal systems of crystallizing magmas on the basis of transport theory and oxygen isotope data: an analysis of the Skaergaard intrusion. J. Petrology 20, 421-486.

Pruess, K (1983) Development of the general purpose simulator MULKOM. In: Earth Sciences Division Annual Report, 1982 LBL-15500, 133-134.

Reed, M. H. (1982) Calculation of multicomponent chemical equilibria and reaction processes in systems involving minerals, gases and an aqueous phase. Geochim. Cosmochim. Acta 46, 513-528. 
Rhee, H.-K., and Amundson, N. R. (1970) An analysis of an adiabatic adsorption column: Part 1. Theoretical development. Chem. Eng. Jour. 1, 241-254.

Rimstidt, J. D. and Barnes H. L. (1980) The kinetics of silica-water reactions. Geochimica et Cosmochimica Acta 44, 1683-1699.

Rubinstein, L. 1. (1971) The Stefan Problem. Trans. Math. Mon., Amer. Math. Soc., 419 pp. Providence.

Valocchi, A. J., Street, R. L. and Roberts, P. V. (1981) Transport of ion-exchanging solutes in groundwater. Chromatographic theory and field simulation. Water Resources Research 17, 1517-1527.

Vaughan, P. J. and Byerlee, J. D. (1984) Mechanism of permeability reduction during flow of hot aqueous solutions through Westerly granite. Trans. American Geophysical Union (Abstract) 65, 1126.

Voller, V. R., Cross M. and Walton P. (1979) Assessment of weak solution techniques for solving Stefan problems. In: Numerical Methods in Thermal Problems Eds. R. W. Lewis and K. Morgan, Pineridge Press, 172-181.

Voller, V. and Cross, M. (1981) Accurate solutions of moving boundary problems using the enthalpy method. Int. J. Heat Mass Transfer 24, 545-556.

Voller, V. and Cross, M. (1983) An explicit numerical method to track a moving phase change front. Int. J. Heat Mass Transfer 26, 147-150.

Walsh, M. P., Bryant, S. L., Schechter, R. S. and Lake, L. W. (1984) Precipitation and dissolution of solids attending flow through porous media. Am. Inst. Chem. Eng. J. 30, 317327.

Wangen, L. E., Stallings, E. A. and Walker, R. D. (1982) The transport of contaminants from energy process waste leachates through subsurface soils and soil components: laboratory experiments. Los Alamos National Laboratory, LA-9406-MS, 39 pp. 
Wangen, L. E. and Stallings, E. A. (1984) Subsurface transport of contaminants from energy process waste leachates. Los Alamos National Laboratory Progress Report, LA-1011PR, 12 pp.

Wolery, T. J. (1979) Calculation of chemical equilibrium between aqueous solution and minerals: the EQ3/6 software package. Lawrence Livermore Laboratory URCL-52658, $41 \mathrm{pp}$. 


\section{FIGURE CAPTIONS}

Fig. 1. - Aqueous concentration of solute species A and B plotted as a function of distance after a time of $1 \times 10^{5} \mathrm{sec}$ has elapsed. The solid lines refer to the exact solution to the reaction-diffusion equations. The circles represent the result of a numerical implicit finite difference calculation with a grid spacing of $\Delta x=0.02 \mathrm{~cm}$ and a time step $\Delta t=10^{3} \mathrm{sec}$. The calculations were carried out with a diffusion coefficient of $D=1 \times 10^{-6} \mathrm{~cm}^{2} / \mathrm{sec}$, porosity $\phi=0.2$, a solid phase molar volume of $0.1 \mathrm{~cm}^{3} / \mathrm{mole}$ and initial volume fraction $\phi_{s}(x, t=0)=0.1$, and an equilibrium constant of $K_{e q}=1$. The quantity $\times$ denotes the ratio of the initial solid volume fraction to porosity divided by the solid molar volume. The kink in the concentration profiles marks the position of the dissolution front, to the left of which complete dissolution of the solid phase has occurred.

Fig. 2. - Position of the reaction front located at the kink in the solute concentration shown in Fig. 1. plotted as a function of time. The dashed curve refers to the exact solution and the solid line represents the numerical finite difference solution. The same parameters were used as in Fig. 1.

Fig. 3. - Geometry used in the counter diffusion problem. The two solute species A and B diffuse from opposite ends of a porous column, reacting to form the solid precipitate $C_{(s)}$ shown by the hatched region.

Fig. 4. - The position of the reaction zone boundary plotted as a function of time for the reaction $A+B \neq C_{(s)}$ illustrating the behavior of the transient regime and the approach to steady-state. The points labeled $a, b$ and $c$ are discussed in the text. The parameters used in the calculation were equal diffusion coefficients for each solute species with a value of $10^{-6} \mathrm{~cm}^{2} / \mathrm{sec}$, equilibrium constant $K=10^{-6}$ and symmetrical boundary conditions of 1 mole/liter for each species. 
Fig. 5. - Position of the reaction zone boundaries for the asymmetrical boundary conditions $C_{\AA}^{0}=2$ and $C_{\mathrm{B}}^{0}=0.5$ moles/liter versus time. The same value for the diffusion coefficient was used as in the previous example. The equilibrium constant used in the calculation had a value of $K=10^{-2}$.

Fig. 6. - Concentration profiles for the system considered in Fig. 5. versus distance for two different times, the dot-dashed curve corresponding to $6 \times 10^{4} \mathrm{sec}$ and the solid curve to $4.3 \times 10^{6} \mathrm{sec}$ corresponding to steady-state.

Fig. 7. - The precipitation rate for the system considered in Fig. 5.

Fig. 8. - The positions of the reaction zone boundaries versus time for the case of two solid phases, the upper curves referring to solid $C_{1(s)}$ and the lower curves to solid $C_{2(s)}$. The parameters used the calculation were equal diffusion coefficients with the value $10^{-6} \mathrm{~cm}^{2} / \mathrm{sec}$, equilibrium constants $K_{1}=10^{-4}$ and $K_{2}=10^{-5}$ and symmetrical boundary conditions of 1 mole/liter. Invariant points occur where the inner boundaries touch.

Fig. 9. - Results of an implicit finite difference calculation for the aqueous concentration profiles of solute species $C_{1}, C_{2}, C_{3}$ and $C_{4}$ plotted as a function of distance at $t=9 \times 10^{4} \mathrm{sec}$. The vertical dashed lines refer to chemical shock fronts. The parameters used in the calculation are a constant fluid flow rate of $1 \mathrm{~m} / \mathrm{yr}$, a diffusion coefficient of $1 \times 10^{-6} \mathrm{~cm}^{2} / \mathrm{sec}$, porosity of $20 \%$, and an initial volume fraction of solid $M_{12}$ of 0.1 .

Fig. 10. - Positions of the reaction zone boundaries corresponding to the vertical dashed lines in Fig. 9, plotted as a function of time.

Fig. 11. - Volume fractions occupied by solids $M_{13}$ and $M_{24}$ at the same time as in Fig. 9 . plotted as a function of distance. 
Fig. 12a. - Position of the reaction zone boundaries plotted as a function of time for the secondary minerals pyrophyllite, muscovite, kaolinite and gibbsite, resulting from the irreversible reaction of $\mathrm{K}$-feldspar at $100^{\circ} \mathrm{C}$. Parameters used in the calculation were a fluid flow rate of $1 \mathrm{~m} / \mathrm{yr}$, a diffusion coefficient of $1 \times 10^{-6} \mathrm{~cm}^{2} / \mathrm{sec}$, a specific reactive surface area of $30 \mathrm{~cm}^{-1}$, a porosity of $20 \%$, and an initial volume fraction of $\mathrm{K}$-feldspar of 0.1 . The $\mathrm{pH}$ of the initial solution was 6 , with a concentration of $\mathrm{K}^{+}$of 0.01 mole/liter. A pure $\mathrm{HCl}$ solution of pH 5 was injected into one end of a porous column containing the K-feldspar.

Fig. 12b. - The same as Fig. 12a. but for a longer time span.

Fig. 13. - The volume fractions of muscovite and gibbsite plotted as a function of distance for times $3 \times 10^{5}, 4 \times 10^{5}$ and $5 \times 10^{5} \mathrm{sec}$. The boundary separating the gibbsite-muscovite zones remains essentially constant as internal precipitation takes place within each zone. The same parameters were used in the calculation as in Fig. 12a.

Fig. 14. - The pH plotted as a function of distance at the indicated times for the calculation described in Fig. 12a.

Fig. 15. - The aqueous solution compositions along the flow path for the system described in Fig. 12a. plotted on an activity diagram. The solid curves denote the reaction paths at the indicated times, the left end point of each curve corresponding to the composition of the fluid just inside the inlet and the common right end point corresponding to the initial fluid composition. The dashed curve refers to a closed system calculation with the same initial solution as the injected solution used in the open system calculation.

Fig. 16. - The reaction rate for K-feldspar plotted as a function of distance for the system described in Fig. 12a. A negative value for the rate indicates dissolution, and a positive value precipitation. 
Fig. 17a. - Concentration profiles computed with the computer code MCCTM for solute species involving ion-exchange of $\mathrm{Na}^{+}$and a hypothetical cation $\mathrm{M}^{3+}$ plotted as a function of distance after a time of 5 years has elapsed. The initial conditions consisted of a $0.01 \mathrm{M}$ $\mathrm{NaCl}$ solution in equilibrium with a solid substrate with an exchange capacity of 10 . The injected solution consisted of $0.001 M \mathrm{M}_{2} \mathrm{~L}_{3}$ where $\mathrm{L}=$ denotes a hypothetical ligand, in addition to $0.01 \mathrm{M} \mathrm{NaCl}$. The parameters used in the calculation consisted of a fluid flow rate of $1 \mathrm{~m} / \mathrm{yr}$, a diffusion coefficient of $10^{-5} \mathrm{~cm}^{2} / \mathrm{sec}$ and an ion-exchange selectivity coefficient of 1 .

Fig. 17b. - Reaction rate for $\mathrm{M}^{3+}$ - Na exchange computed with the same parameters used in Fig. 17a.

Fig. 18a. - Concentration profiles for solute species using the same parameters as in Fig. 17a. with the addition of ion complexes $\mathrm{NaL}^{-}$and $\mathrm{ML}^{+}$.

Fig. 18b. - Same as Fig. 18a. except that an injected solution of $0.01 M \mathrm{M}_{2} \mathrm{~L}_{3}$ was used.

Fig. 19a. - Same as Fig. 18a. with the dissociation constant for $\mathrm{ML}^{+}$replaced by $10^{6}$.

Fig. 19b. - Same as Fig. 18a. with the dissociation constant for $\mathrm{ML}^{+}$replaced by $10^{8}$.

Fig. 20. - Same as Fig. 18a with the addition of $0.01 \mathrm{M} \mathrm{H}_{2} \mathrm{SO}_{4}$ to the feed solution.

Fig. 21. - Profiles for $\mathrm{pH}, \mathrm{OH}^{-}, \mathrm{HSO}_{4}^{-}$and $\mathrm{MOH}^{++}$corresponding to Fig. 20.

Fig. 22. - Comparison of the mineral volume change obtained upon cooling a hypothetical granite rock composed of quartz, muscovite, microcline and low albite (solid curves) with quartzite (dot-dashed curve) calculated with the computer package EQ3/6. The dashed curve is the sum of the volume changes for granite.

Fig. 23. - Reduction in the flow rate with time for radial fluid flow through a quartzite rock sample in the form of a cylinder with inner radius $1.27 \mathrm{~cm}$, outer radius $3.8 \mathrm{~cm}$ and length $8.9 \mathrm{~cm}$. The outer surface of the sample was held at a temperature of $250^{\circ} \mathrm{C}$ and a pressure 
of 105 bars. The inner surface was at a temperature of $111^{\circ} \mathrm{C}$ and 100 bars. The solid curve is the result of numerical calculations assuming irreversible precipitation of quartz according to the rate law of Rimstidt and Barnes (1980), with an initial porosity of $7 \times 10^{-4}$, an initial permeability of $1.89 \times 10^{-19} \mathrm{~m}^{2}$ and a specific reactive surface area of $7.7 \times 10^{6} \mathrm{~m}^{-1}$. A cubic dependence of permeability on porosity was used in the numerical simulation. The initial and boundary conditions used in the calculations assumed quartz to be in equilibrium with the fluid at the initial and inlet temperatures respectively. The dashed curve is the result of reversible precipitation of quartz. The dots denote the experimental data of Morrow et al. (1985). An excellent fit to the data is obtained for times up to approximately 100 hours during which the flow rate dropped by two orders of magnitude. For longer times a linear behavior was observed experimentally, which was not obtained from the numerical simulation.

Fig. 24. - Change in porosity with distance at the indicated times for irreversible reaction of quartz for the system described in Fig. 23. The maximum change in porosity occurred close to the inlet resulting from competing effects between the reduction in the reaction rate with temperature and the increase in rate due to the progressive supersaturation of the fluid away from the inlet (see next figure).

Fig. 25. - Mass fraction of aqueous $\mathrm{SiO}_{2}$ plotted as a function of distance at the indicated times for the system described in Fig. 23. The dashed curve denotes the local equilibrium value.

Fig. 26. - Change in porosity with distance at the indicated times for reversible reaction of quartz for the system described in Fig. 23.

Fig. 27. - Fluid pressure as a function of distance at the indicated times calculated with the same parameters as described in Fig. 23. for the irreversible reaction of quartz. A sharp drop in pressure occurs across the neck formed by the precipitation of quartz. 

TABLE 1: REACTIONS DESCRIBING THE METASOMATIC ALTERATION OF MICROCLINE

\begin{tabular}{|c|c|c|}
\hline \multicolumn{3}{|c|}{ SYSTEM: $\mathrm{K}_{2} \mathrm{O}-\mathrm{Al}_{2} \mathrm{O}_{3}-\mathrm{SiO}_{2}-\mathrm{HCl}-\mathrm{H}_{2} \mathrm{O} \quad 100^{\circ} \mathrm{C}$} \\
\hline & aqueous complexing reactions & $\log K$ \\
\hline & $\mathrm{H}_{2} \mathrm{O} \rightleftharpoons \mathrm{H}^{+}+\mathrm{OH}^{-}$ & -12.24 \\
\hline & $\mathrm{Al}(\mathrm{OH})^{++} \rightleftharpoons \mathrm{Al}^{3+}+\mathrm{OH}^{-}$ & -2.24 \\
\hline & $\mathrm{Al}(\mathrm{OH})_{4}^{-} \rightleftarrows \mathrm{Al}^{3+}+4 \mathrm{OH}^{-}$ & -16.87 \\
\hline & $\mathrm{H}_{3} \mathrm{SiO}_{4}^{-} \neq \mathrm{OH}^{-}+\mathrm{SiO}_{2}+\mathrm{H}_{2} \mathrm{O}$ & -9.0 \\
\hline \multicolumn{3}{|c|}{ reversible mineral reactions } \\
\hline mineral & reaction & $\log K$ \\
\hline gibbsite & $\mathrm{Al}(\mathrm{OH})_{3}+3 \mathrm{H}^{+} \neq \mathrm{Al}^{3+}+3 \mathrm{OH}^{-}$ & 4.79 \\
\hline kaolinite & $\mathrm{Al}_{2} \mathrm{Si}_{2} \mathrm{O}_{5}(\mathrm{OH})_{4}+6 \mathrm{H}^{+}=2 \mathrm{Al}^{3+}+2 \mathrm{SiO}_{2}+5 \mathrm{H}_{2} \mathrm{O}$ & 2.36 \\
\hline muscovite & $\mathrm{KAl}_{2}\left(\mathrm{AlSi}_{3} \mathrm{O}_{10}\right)(\mathrm{OH})_{2}+10 \mathrm{H}^{+} \neq \mathrm{K}^{+}+3 \mathrm{Al}^{3+}+3 \mathrm{SiO}_{2}+6 \mathrm{H}_{2} \mathrm{O}$ & -2.86 \\
\hline pyrophyllite & $\mathrm{Al}_{2} \mathrm{Si}_{4} \mathrm{O}_{10}(\mathrm{OH})_{2}+6 \mathrm{H}^{+}=2 \mathrm{Al}^{3+}+4 \mathrm{SiO}_{2}+4 \mathrm{H}_{2} \mathrm{O}$ & 6.36 \\
\hline \multicolumn{3}{|c|}{ irreversible mineral reactions } \\
\hline mineral & reaction & $\log k\left(\mathrm{moles} / \mathrm{cm}^{2} \mathrm{sec}\right)$ \\
\hline microcline & $\mathrm{KAlSi}_{3} \mathrm{O}_{8}+4 \mathrm{H}^{+} \neq \mathrm{K}^{+}+\mathrm{Al}^{3+}+3 \mathrm{SiO}_{2}+2 \mathrm{H}_{2} \mathrm{O}$ & $\begin{array}{c}-9.76(\mathrm{pH}) \\
-14.78\end{array}$ \\
\hline
\end{tabular}




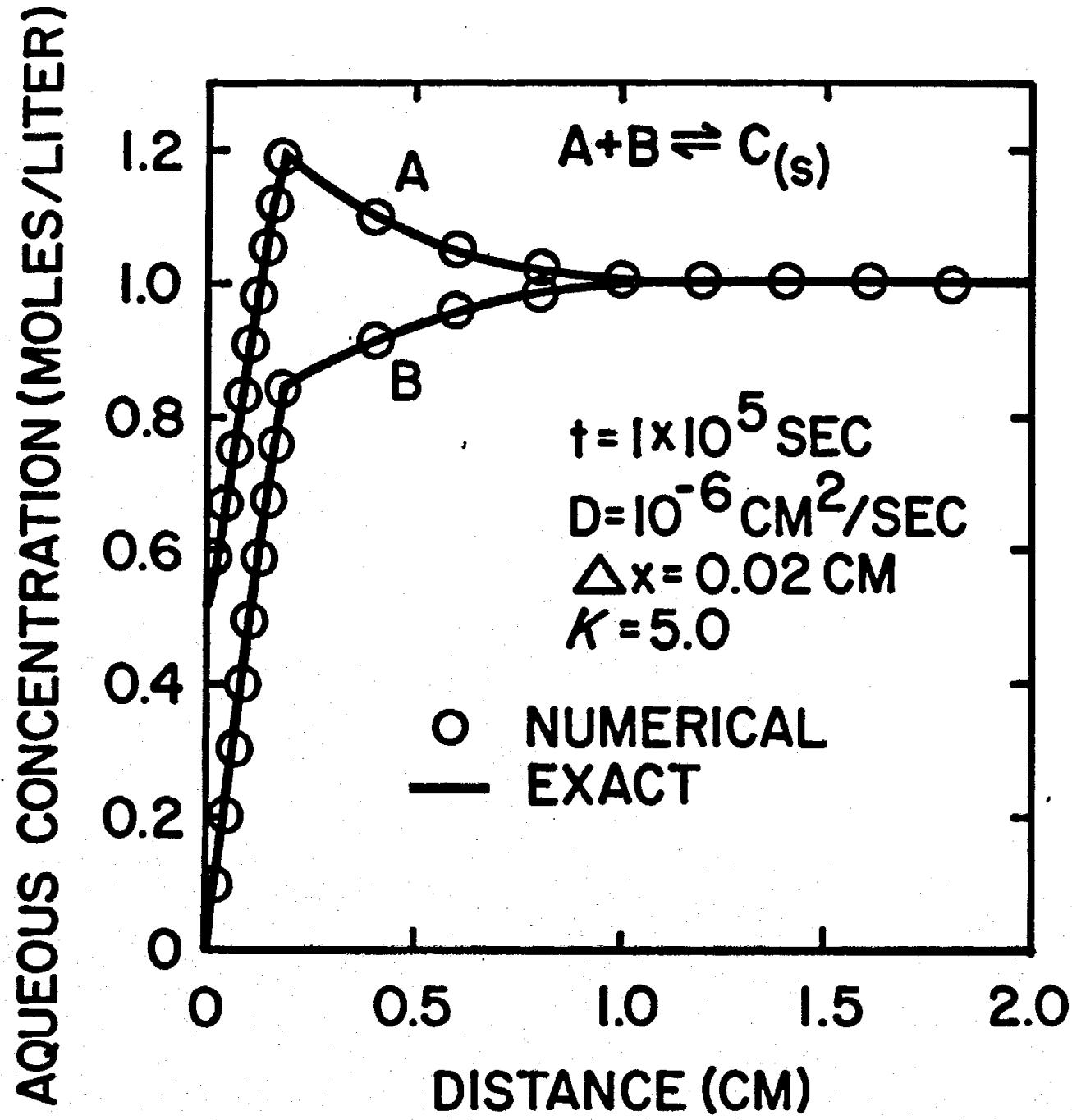

Figure 1 


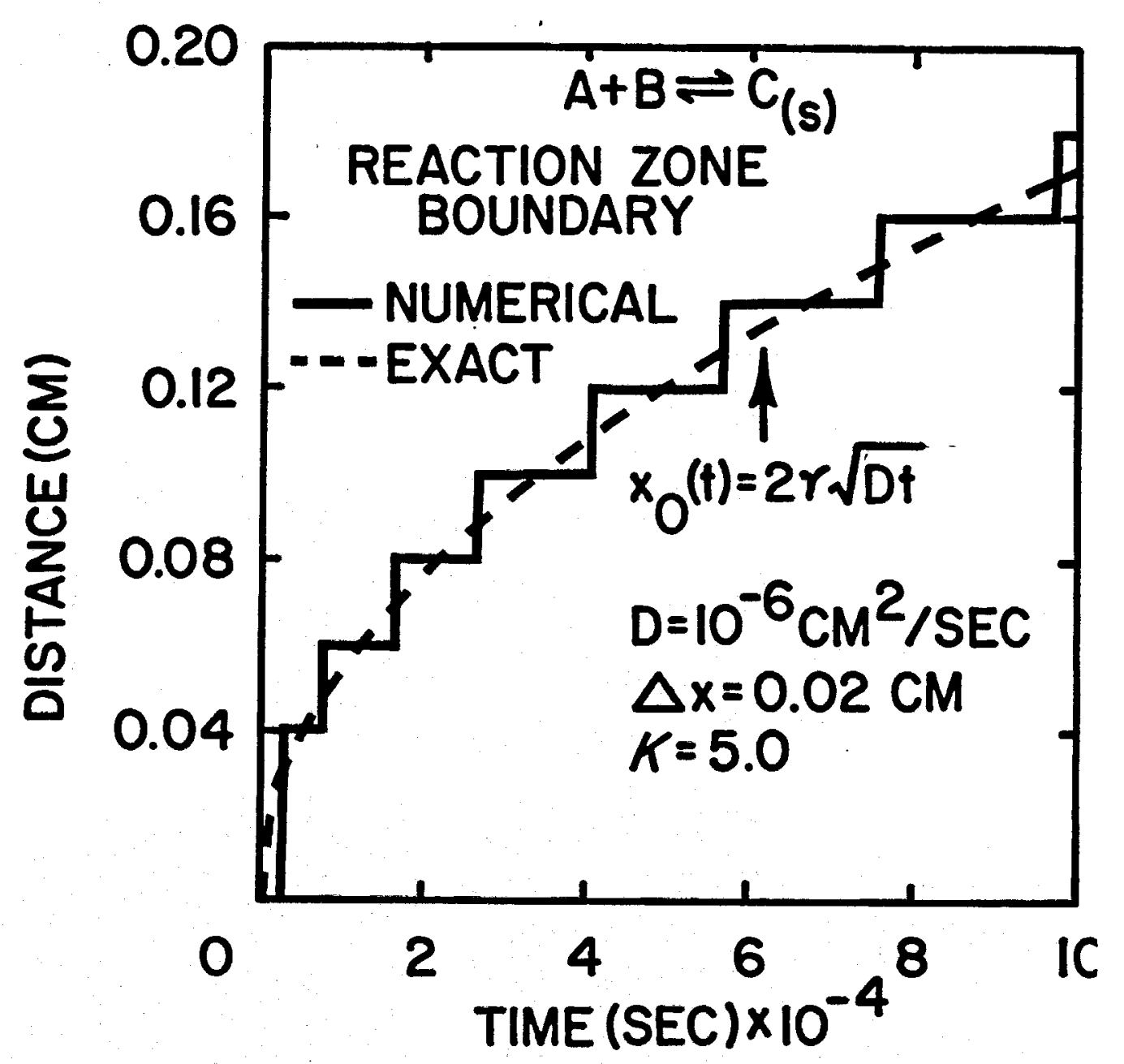

Figure 2 


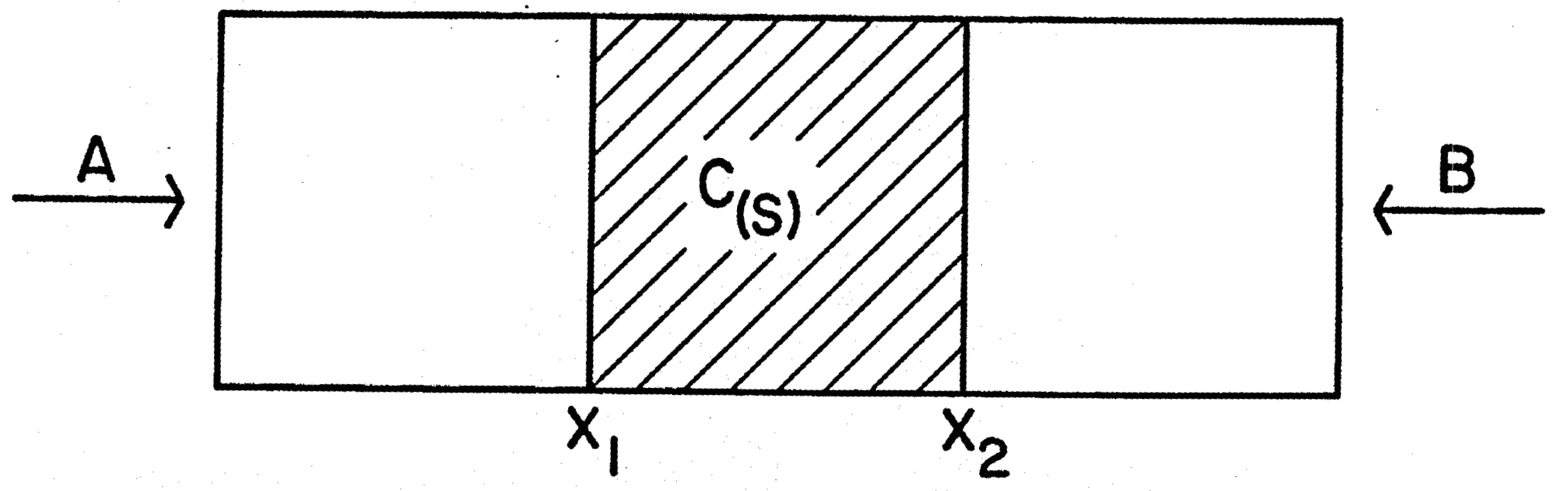

Figure 3 


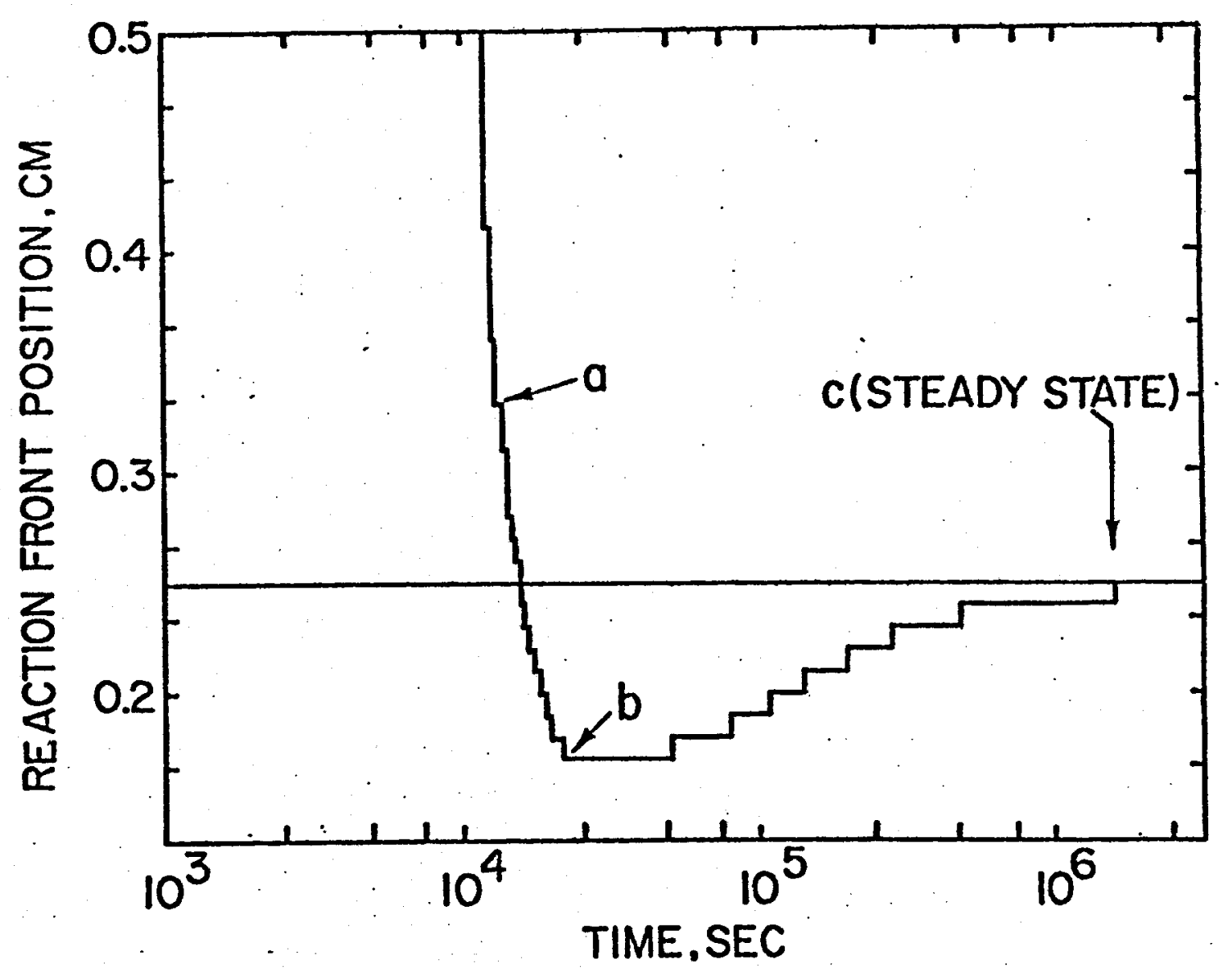

Figure 4 





Figure 6 


\section{LOG REACTION RATE}

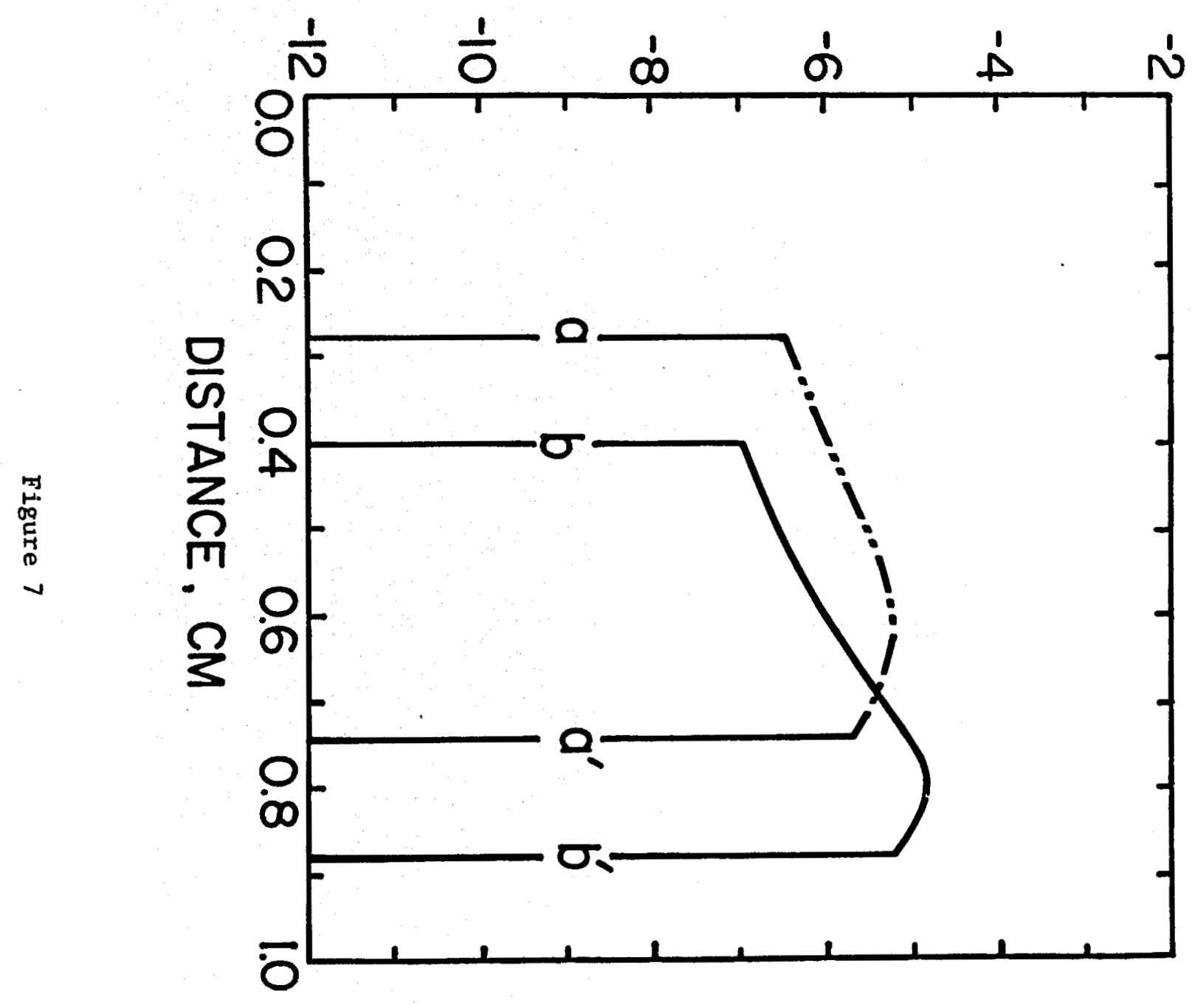




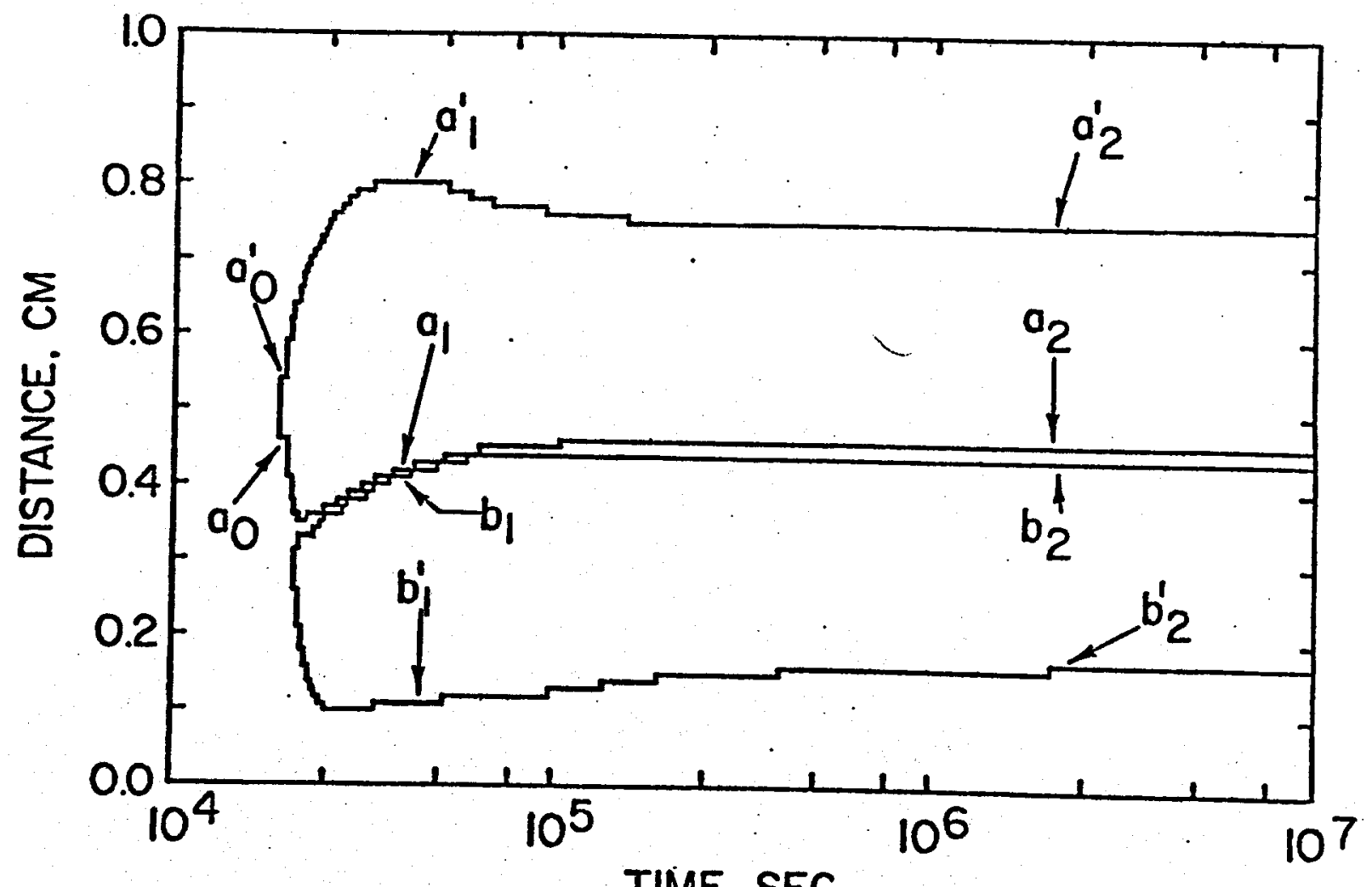

TIME , SEC

Figure 8 


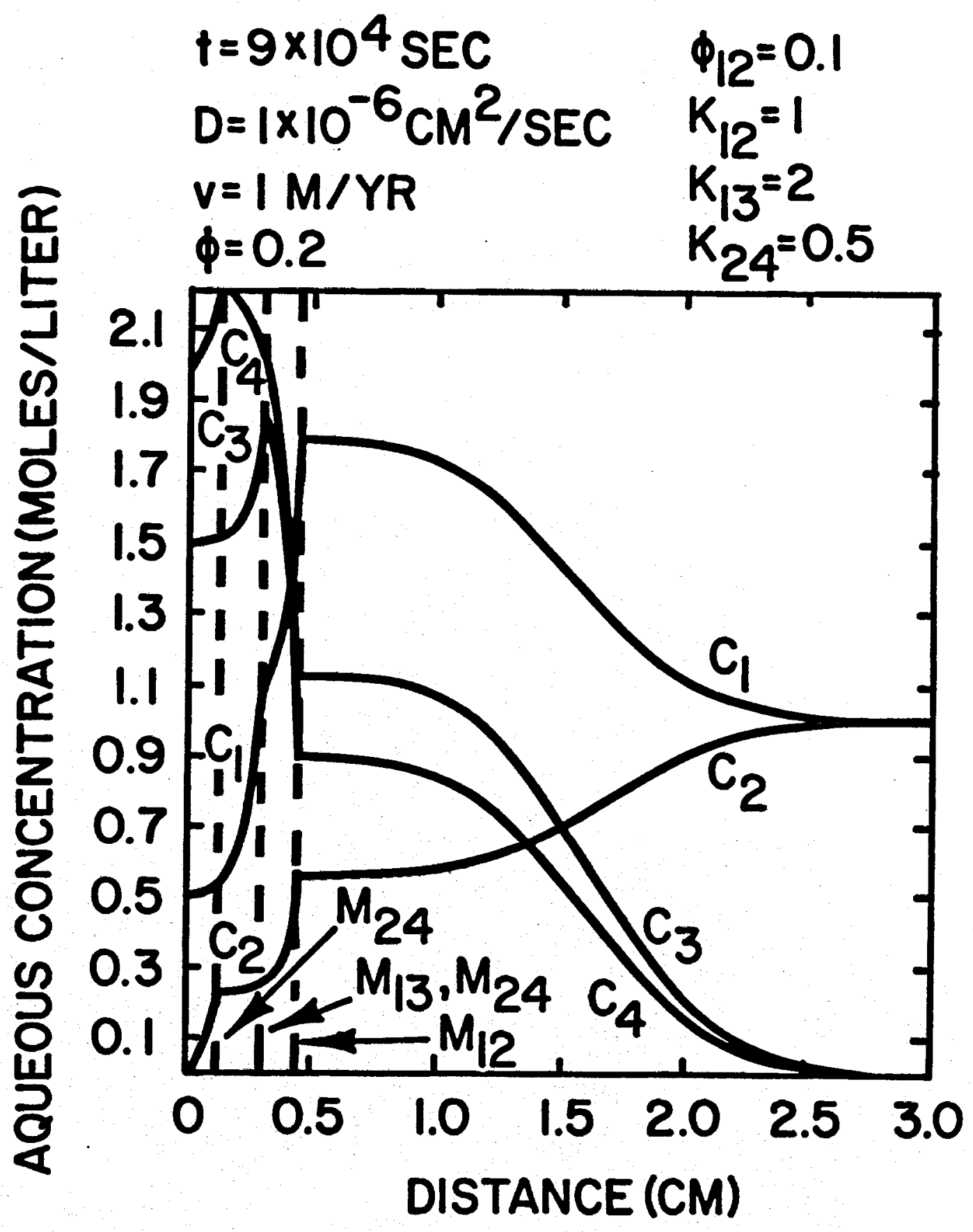

Figure 9 


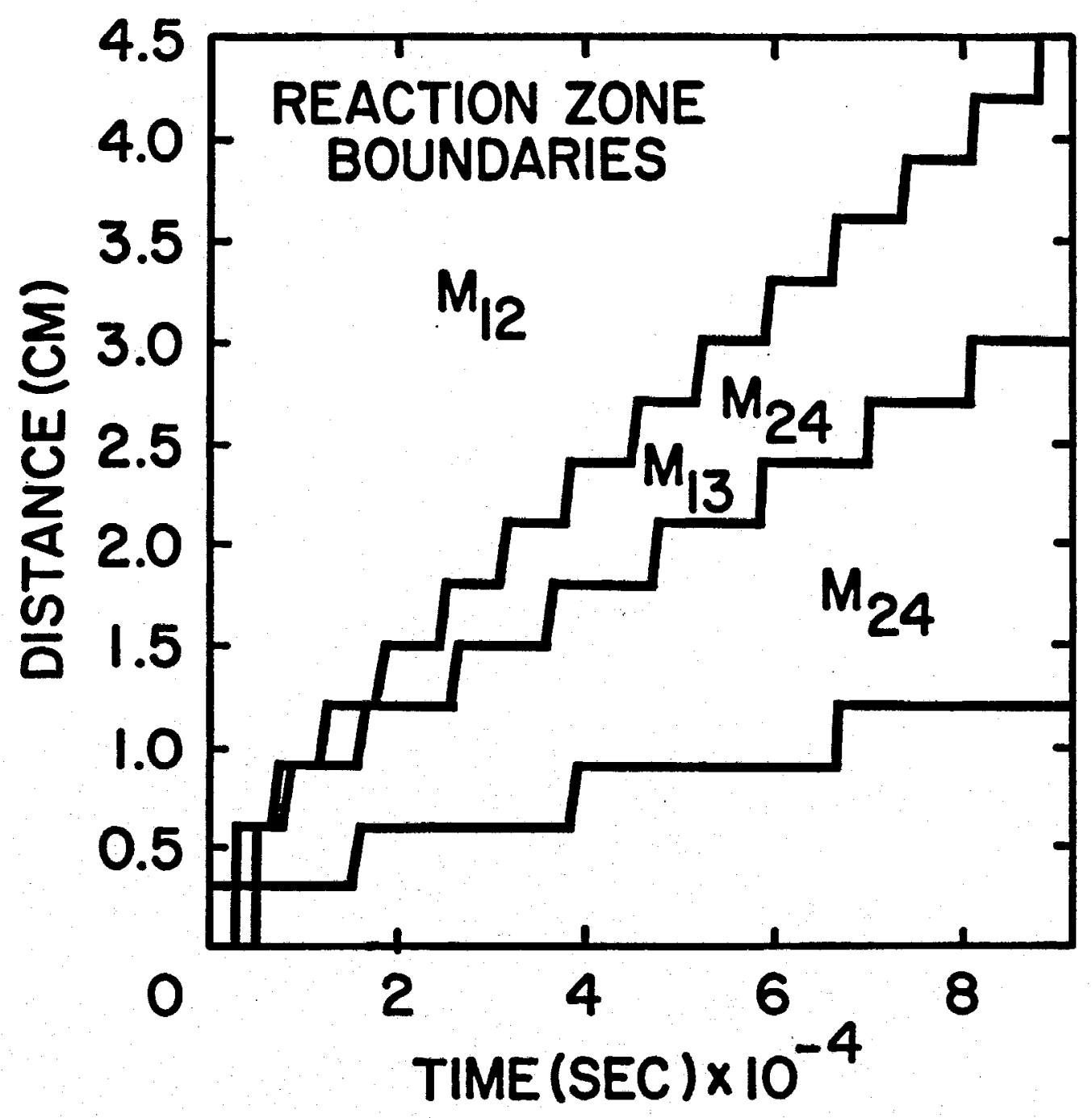

Figure 10 


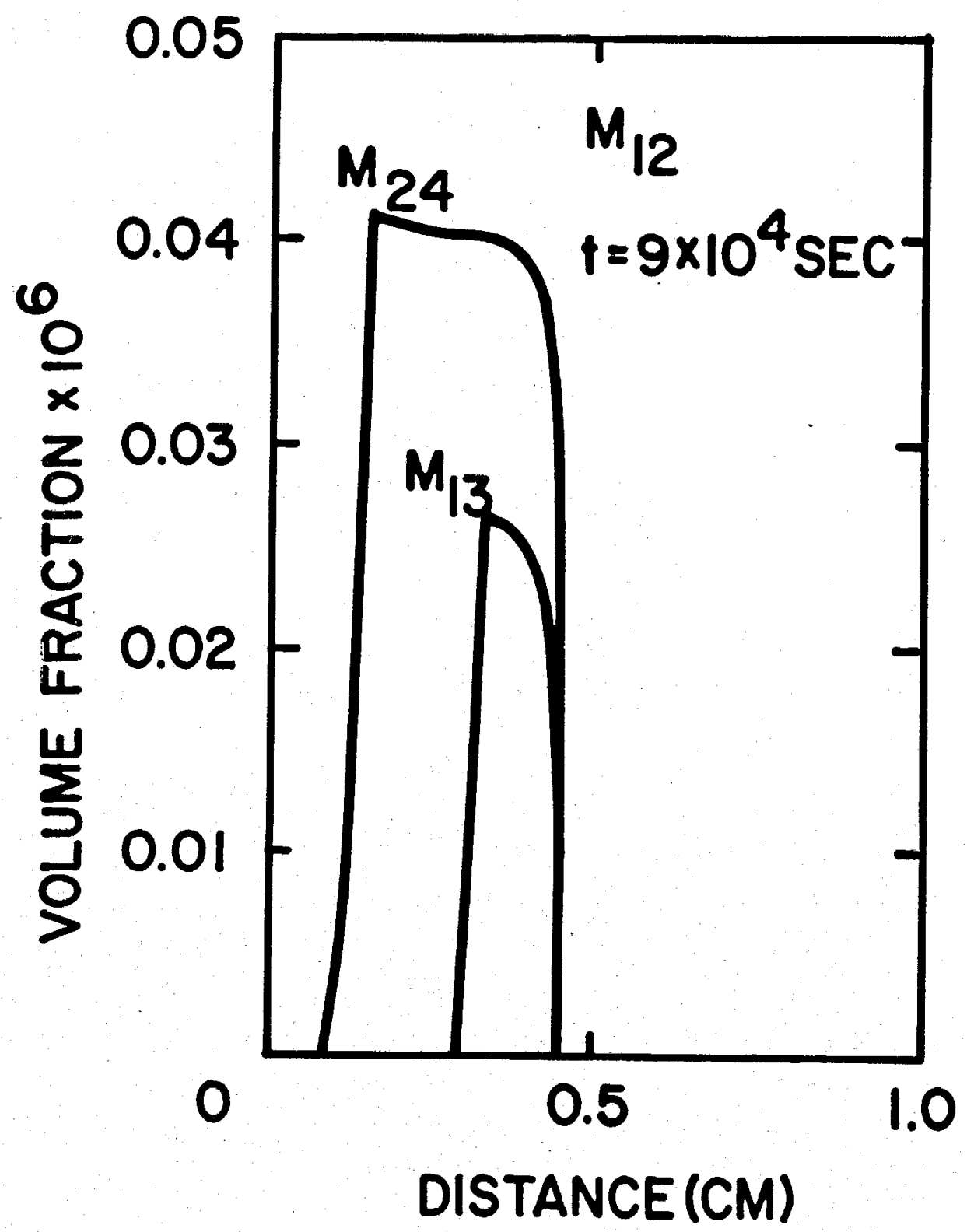

Figure 11 


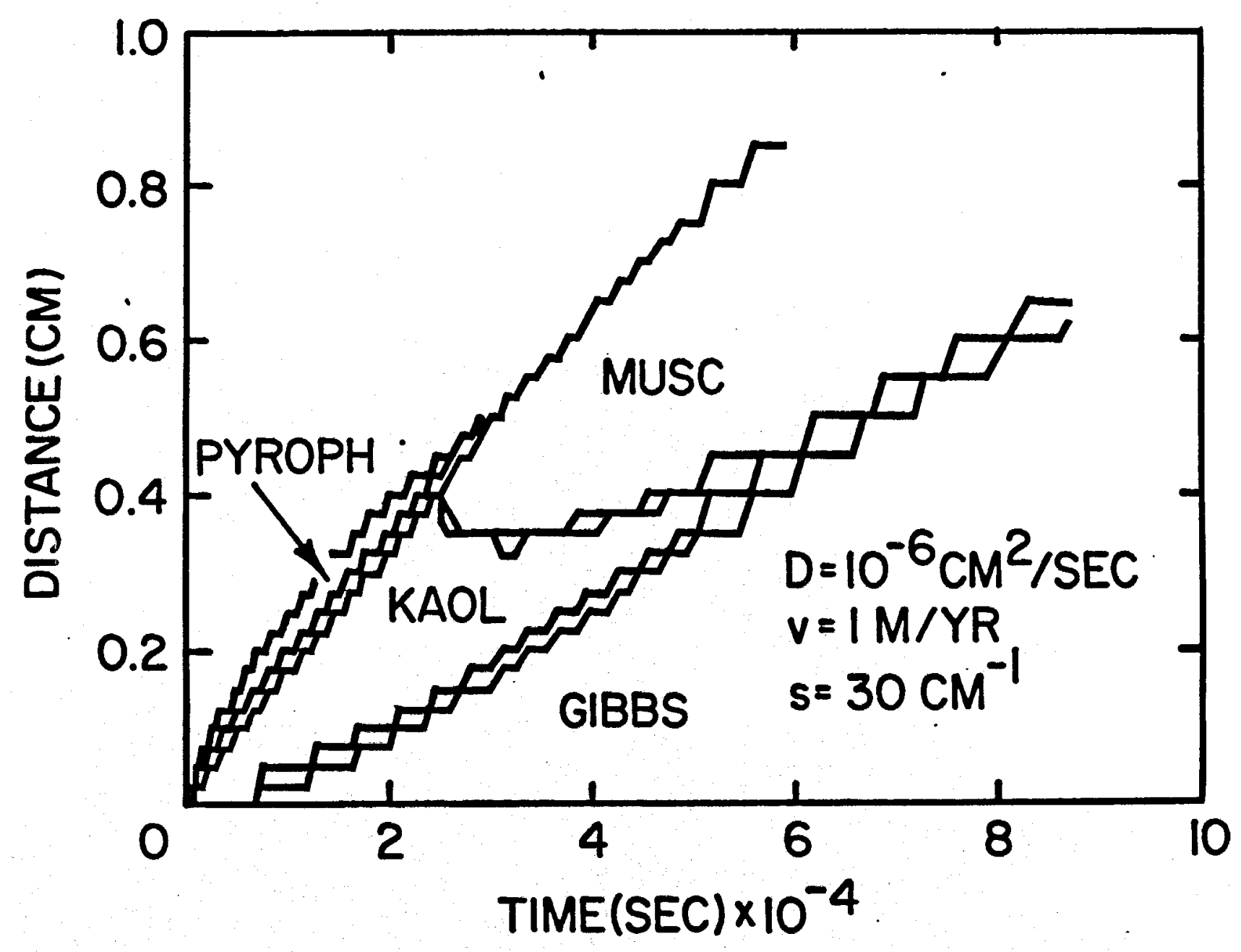

Figure 12a 


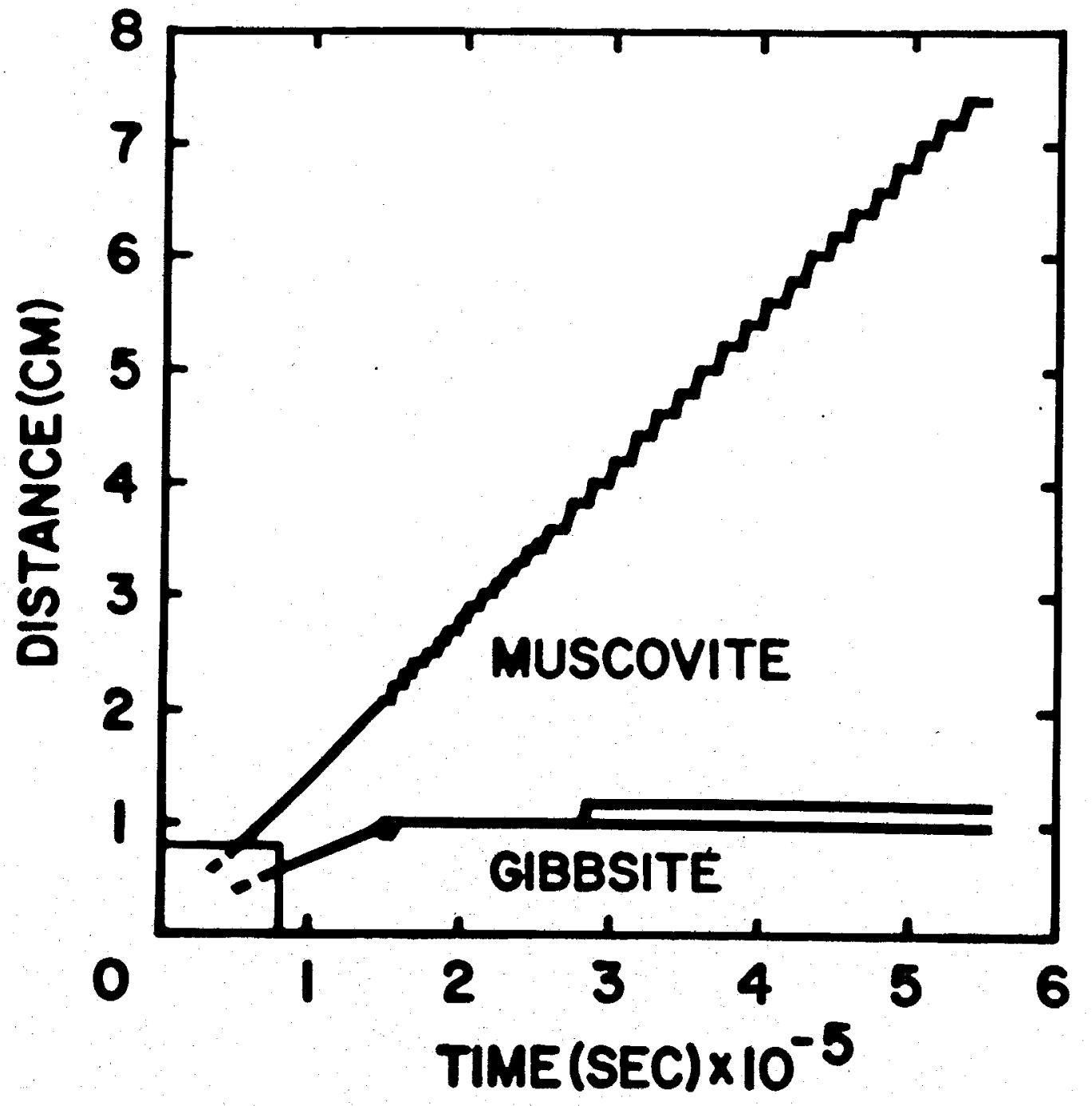

Figure 12b 


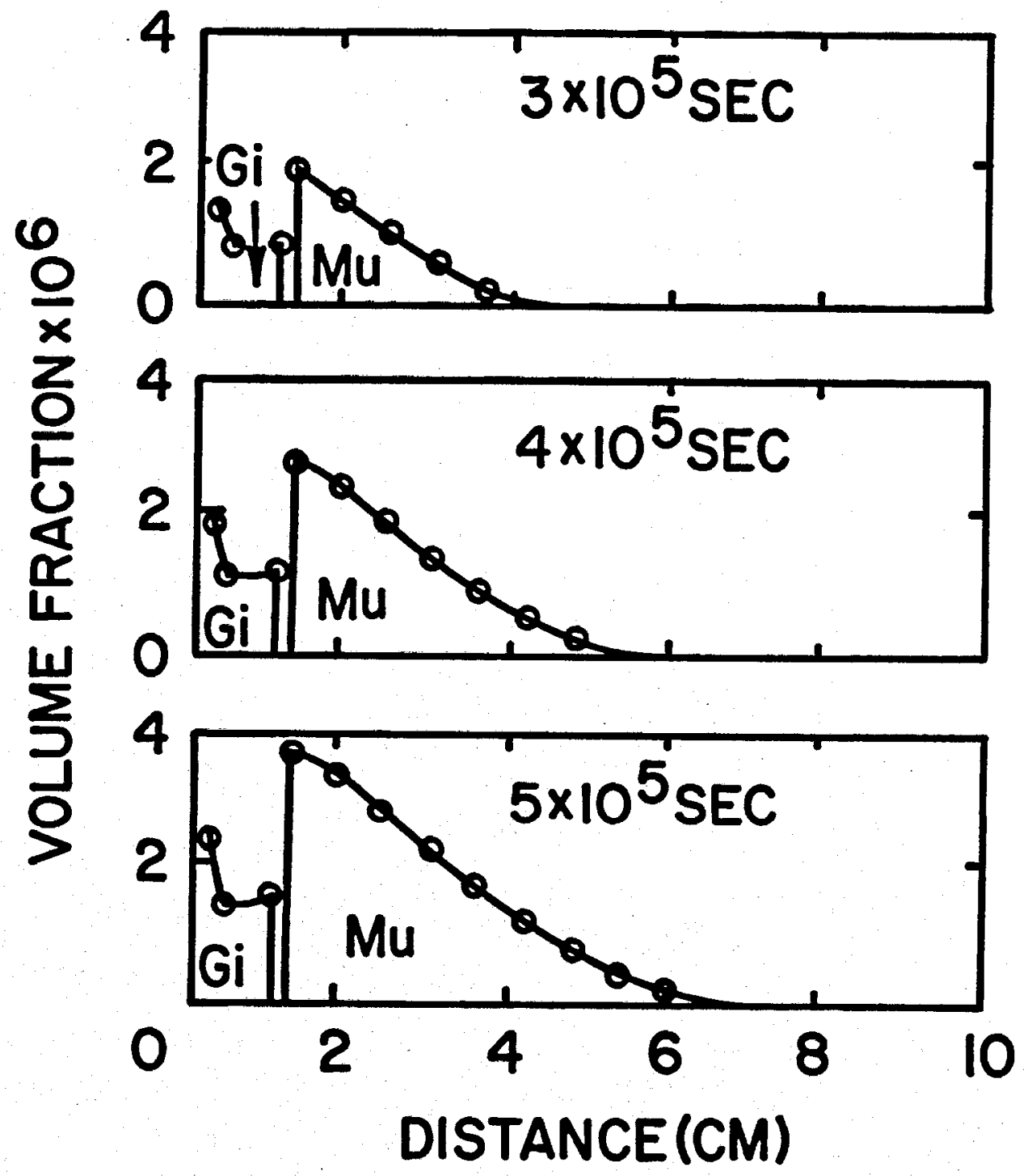

Figure 13 


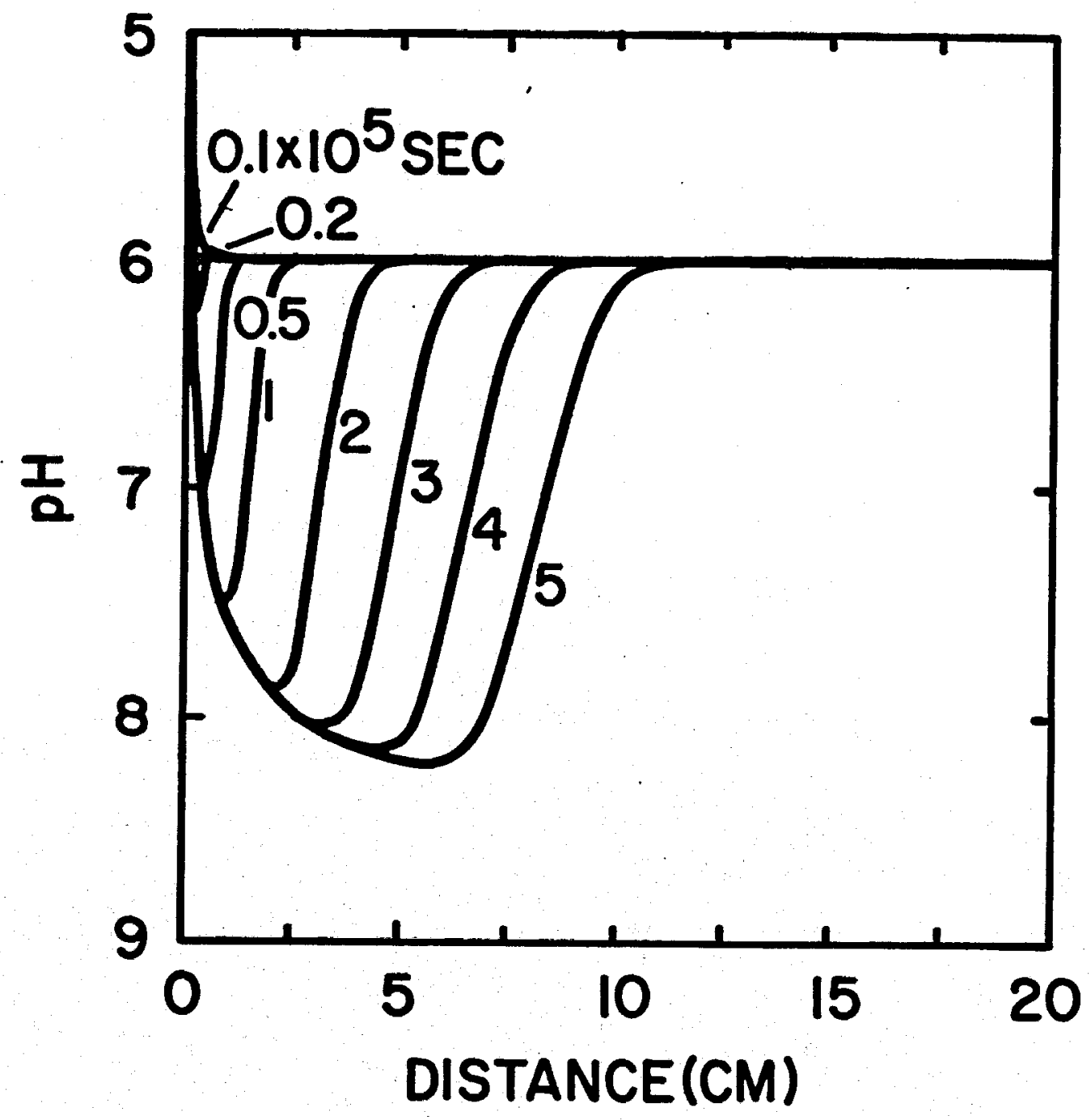

Figure 14 


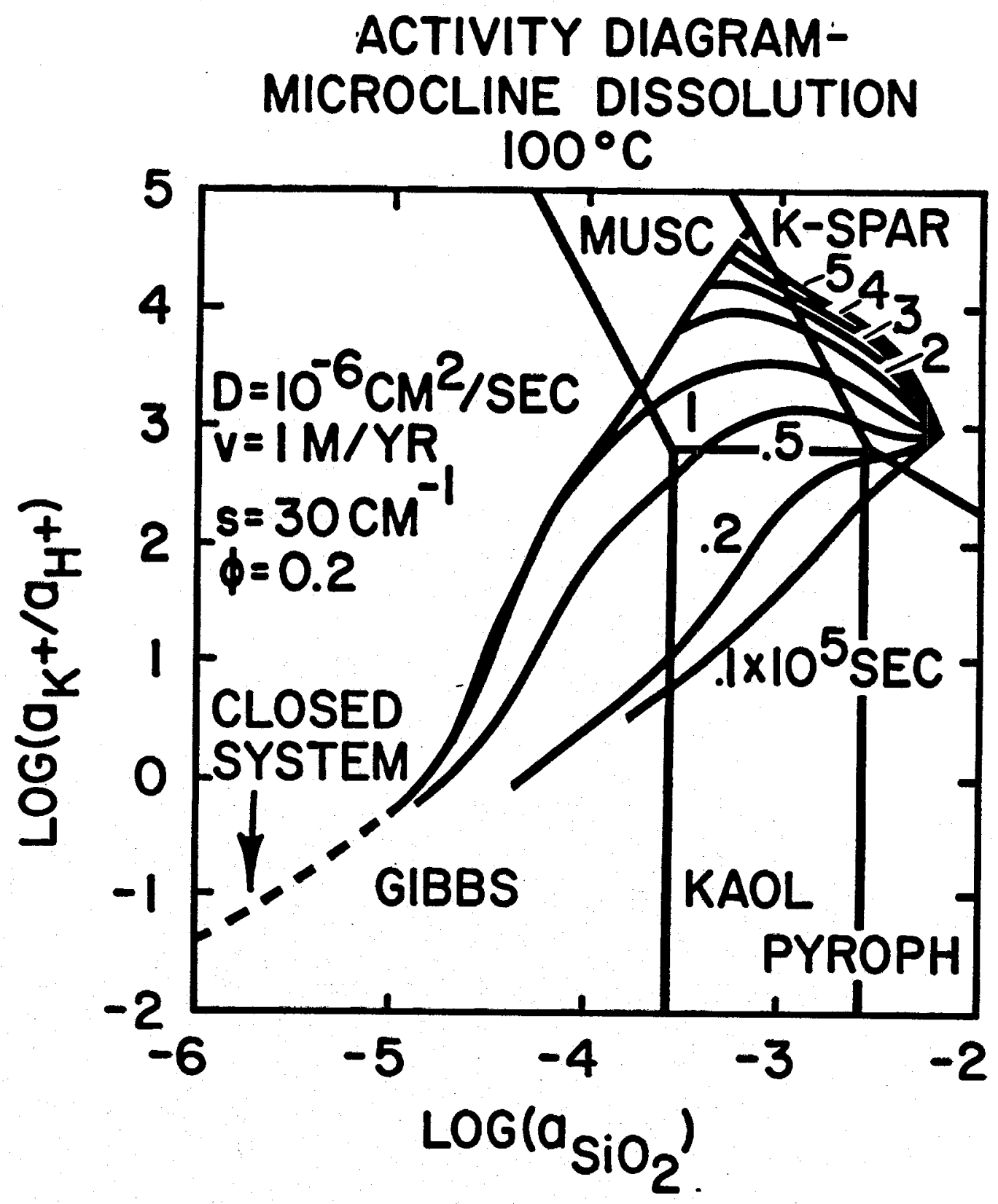

Figure 15 


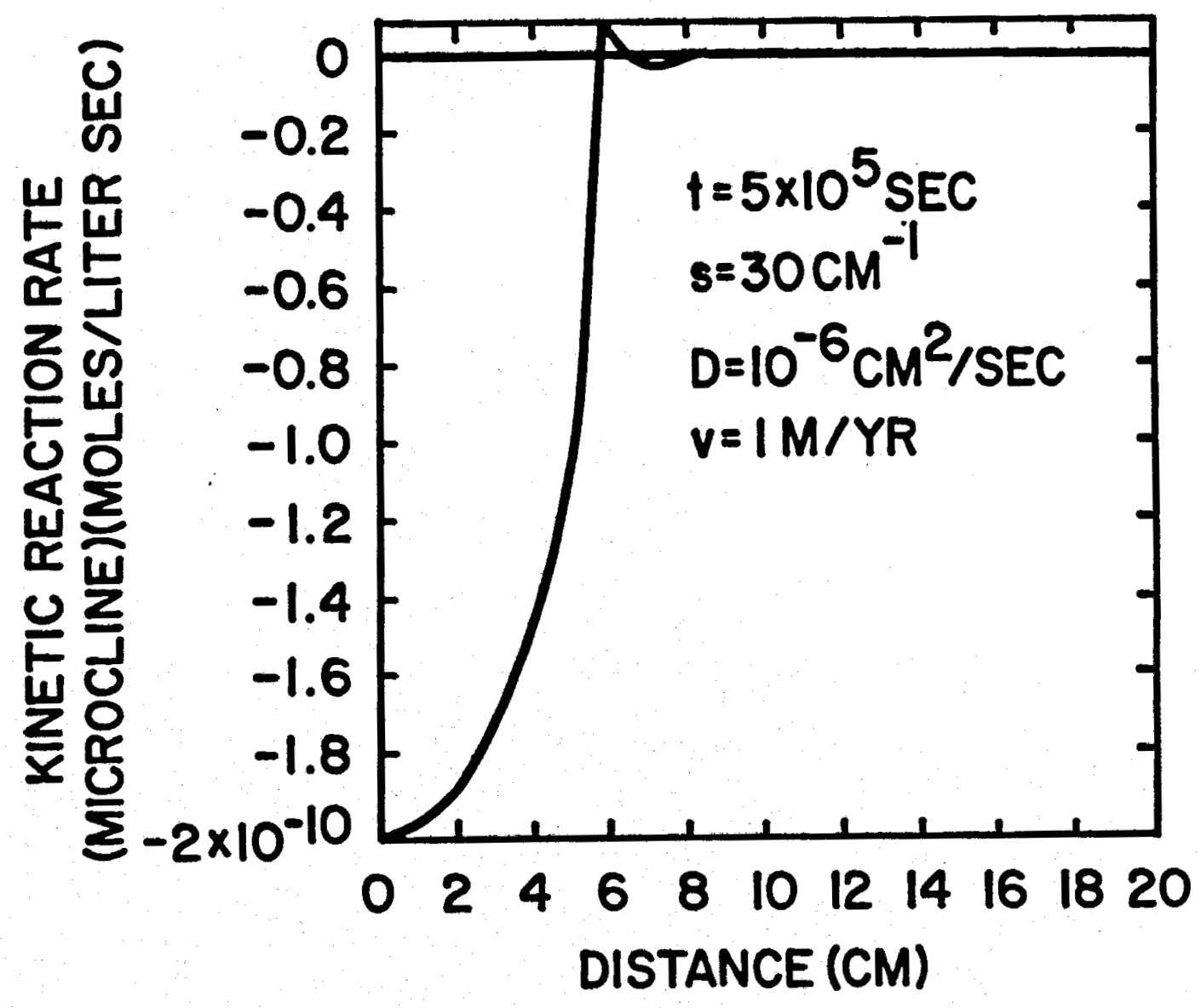

Figure 16 


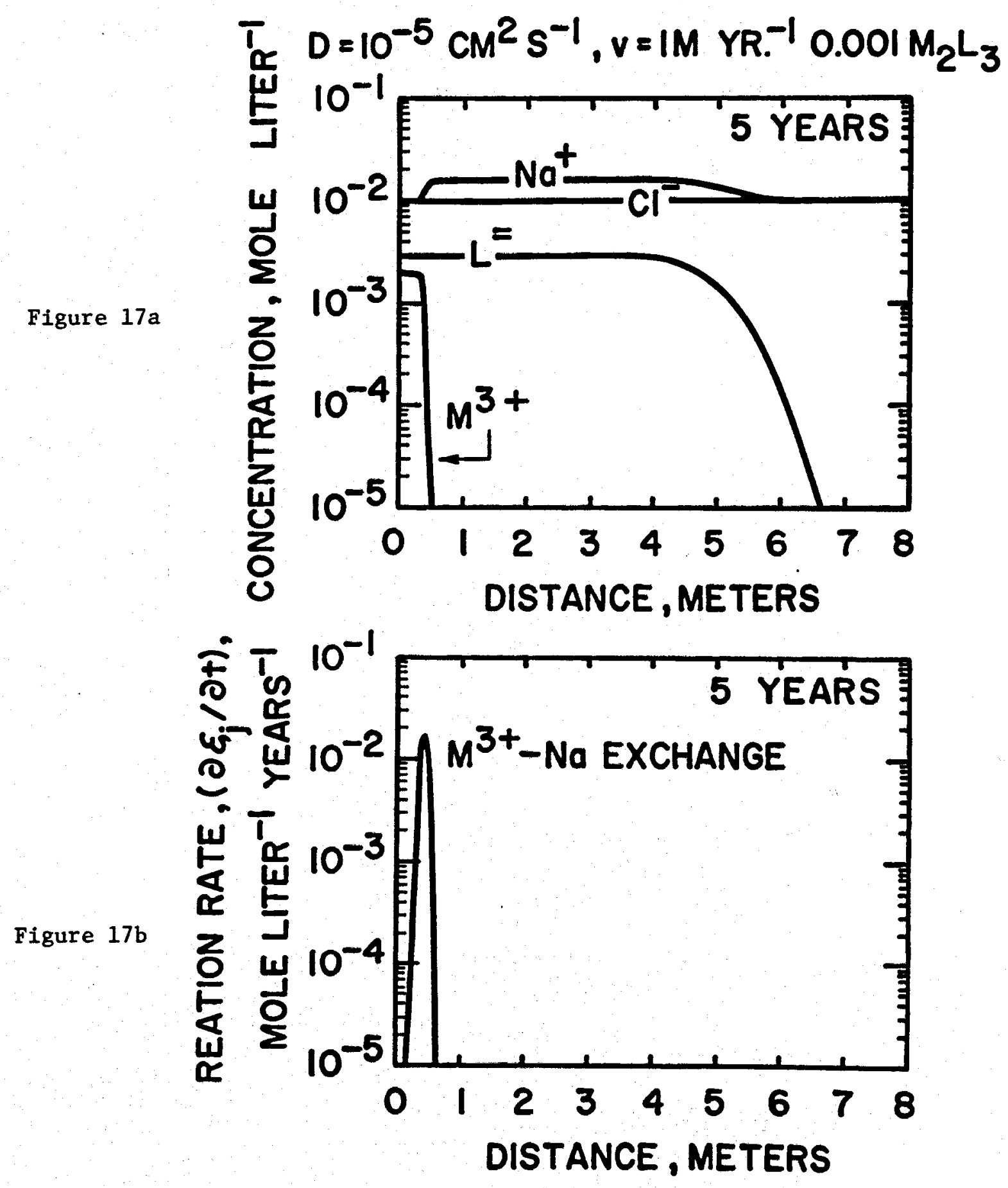




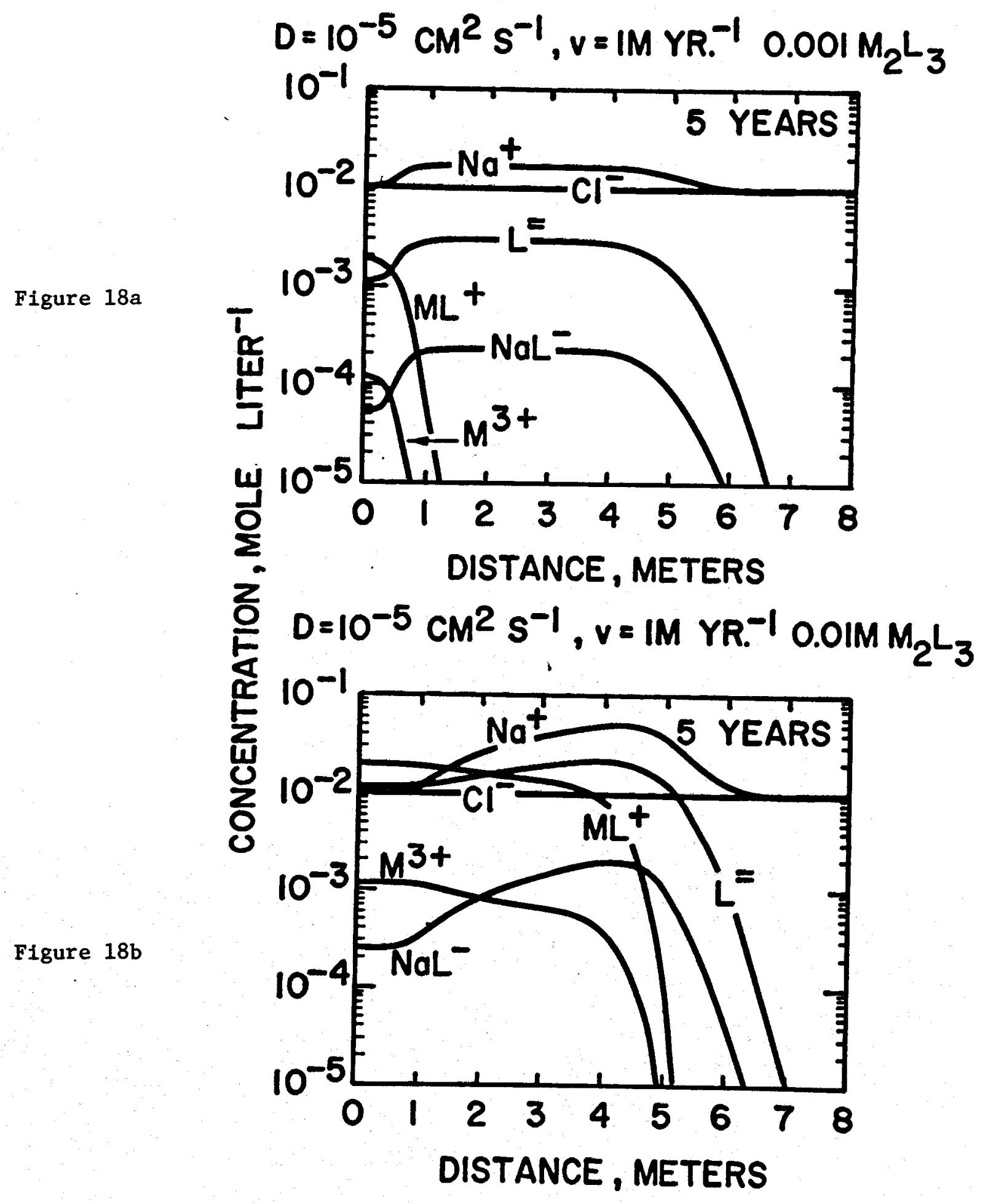




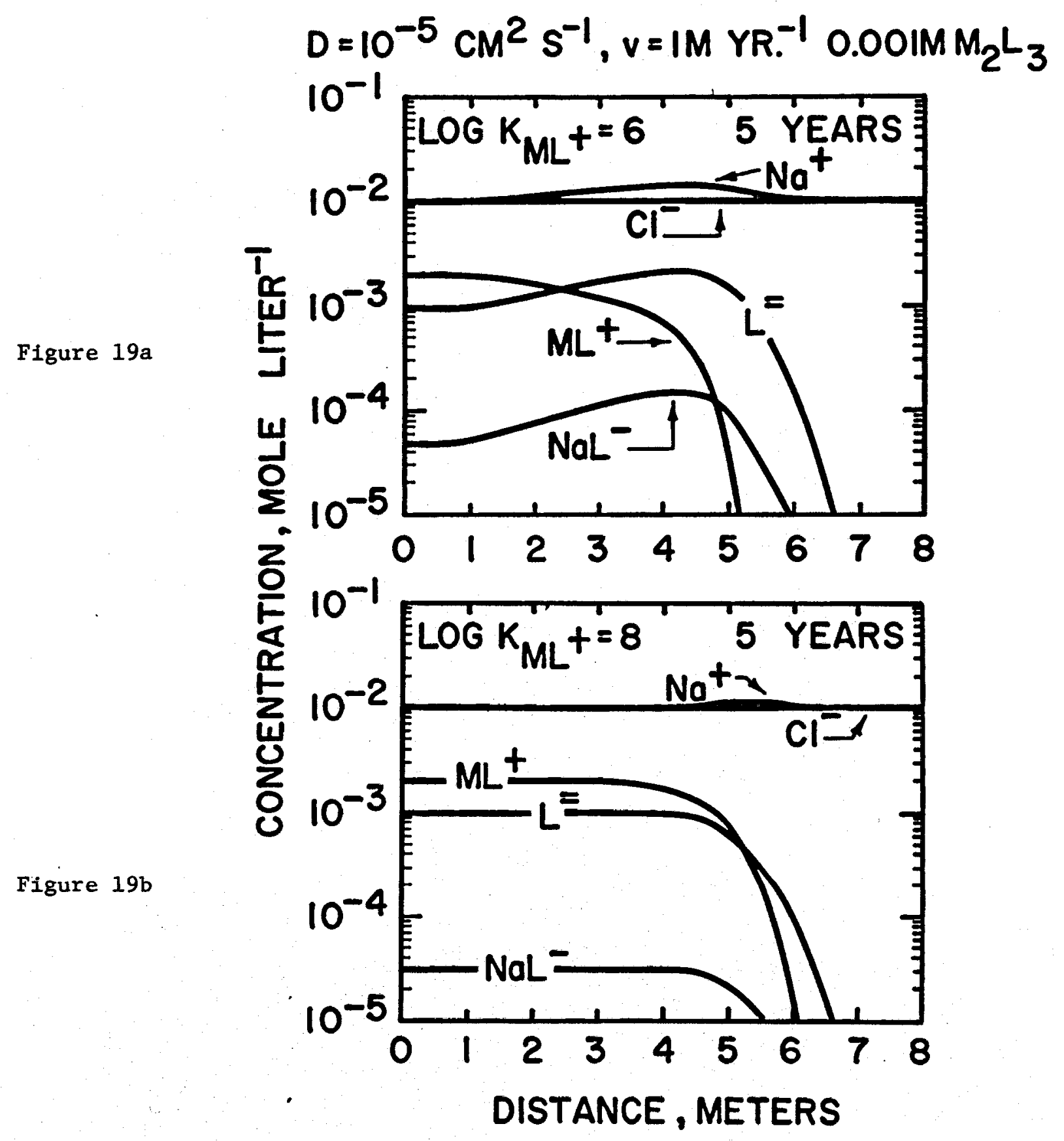




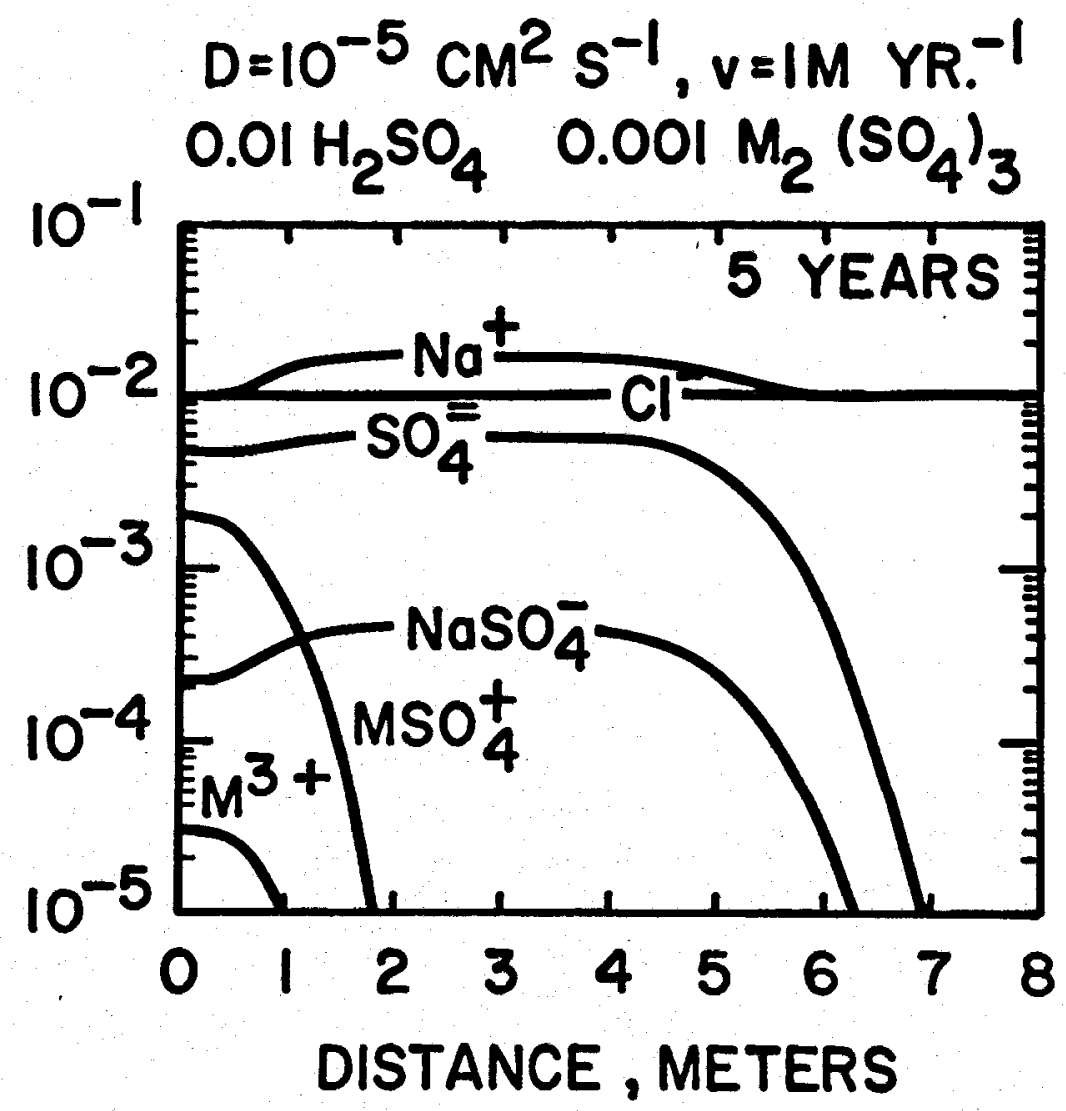

Figure 20 




Figure 21 


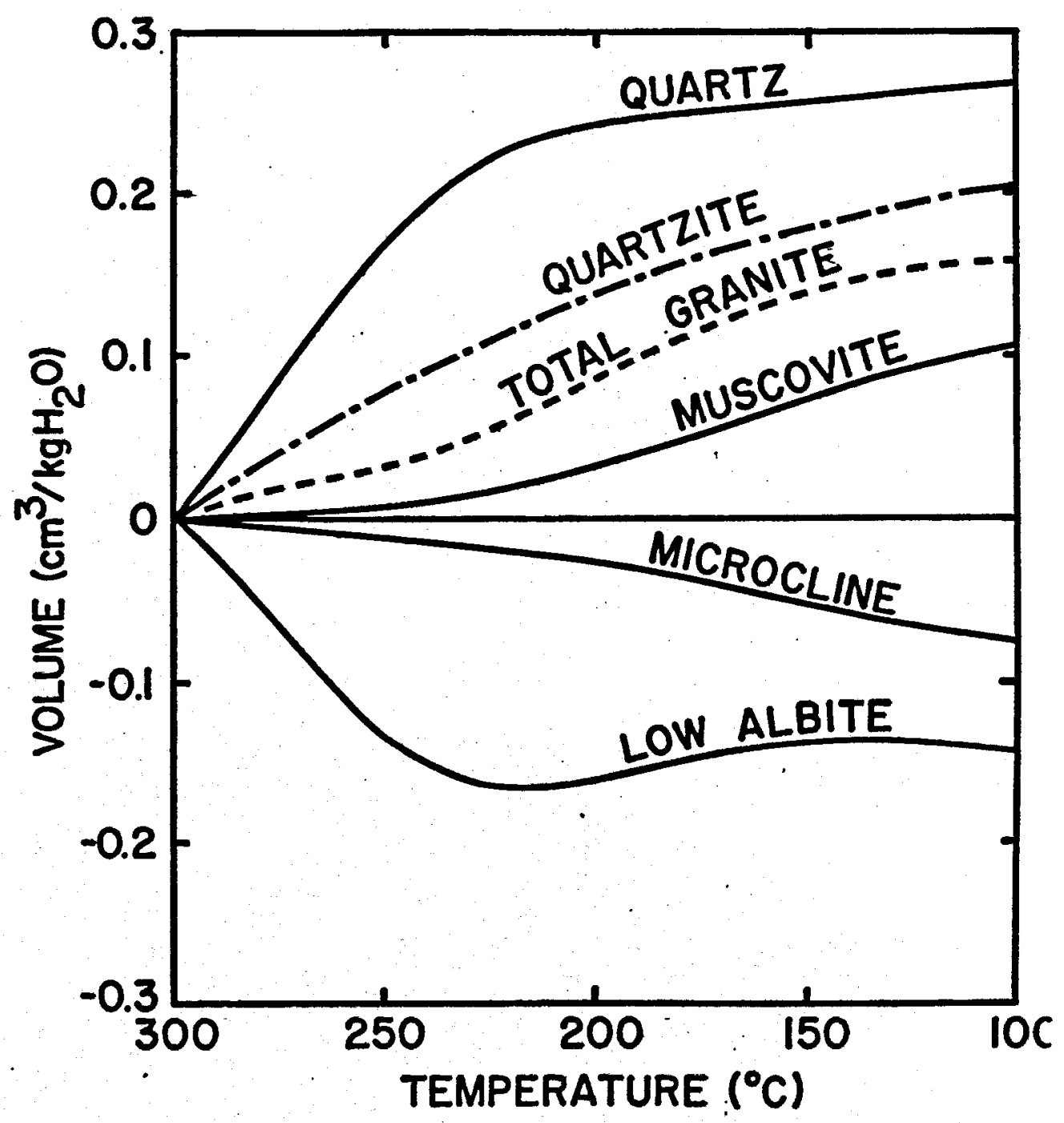

Figure 22 


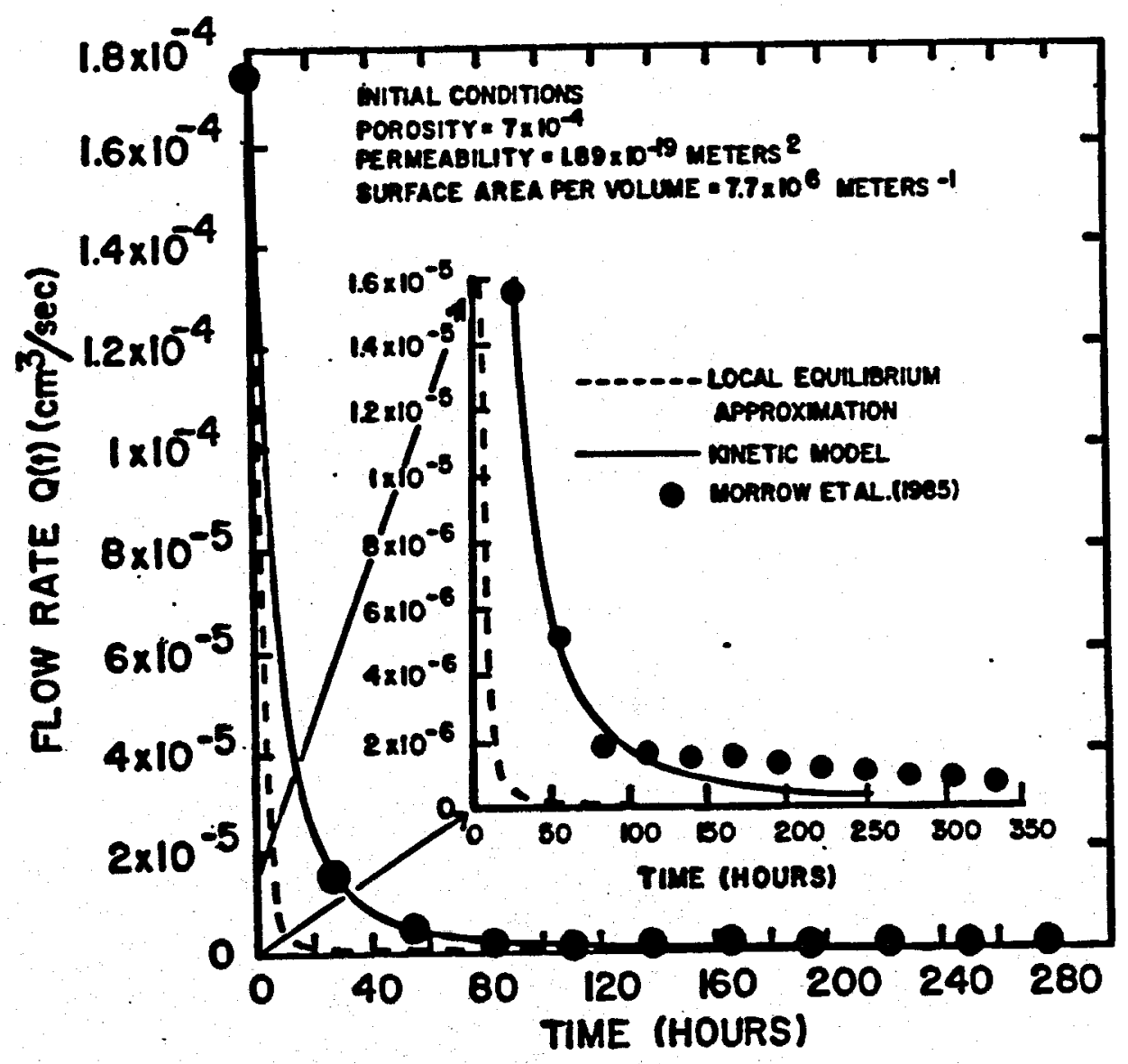

Figure 23 


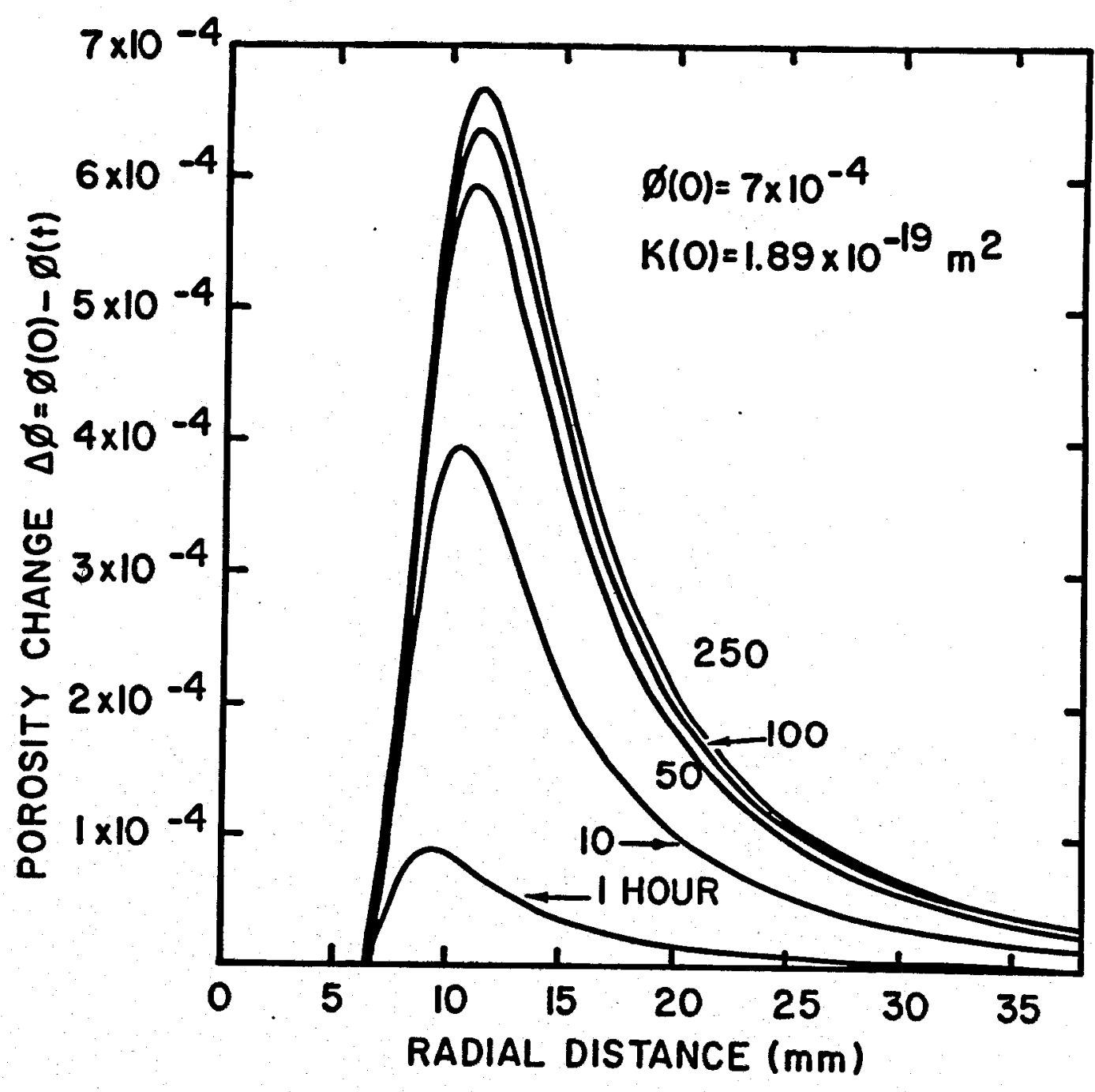

Figure 24 


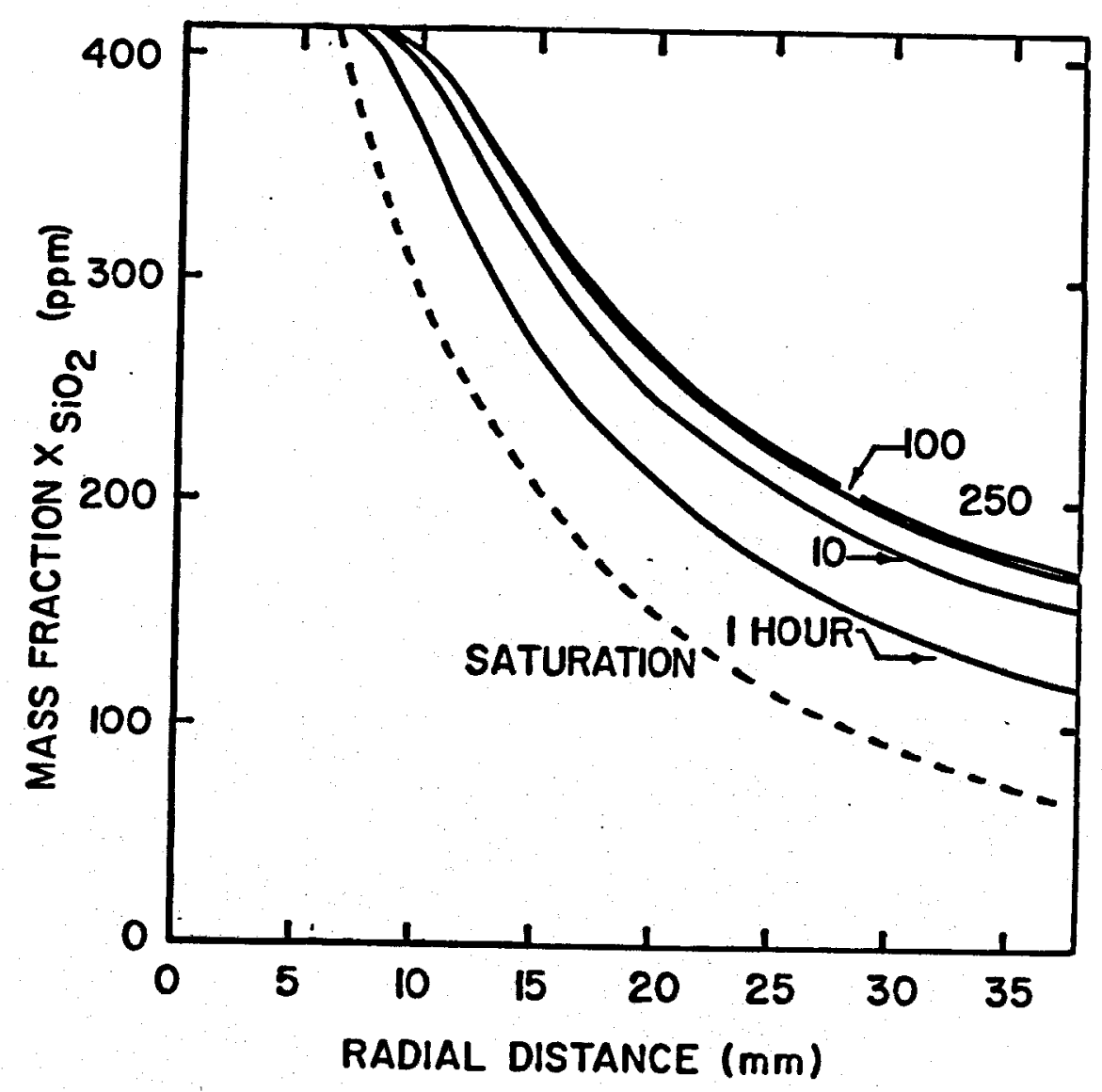

Figure 25 




Figure 26 


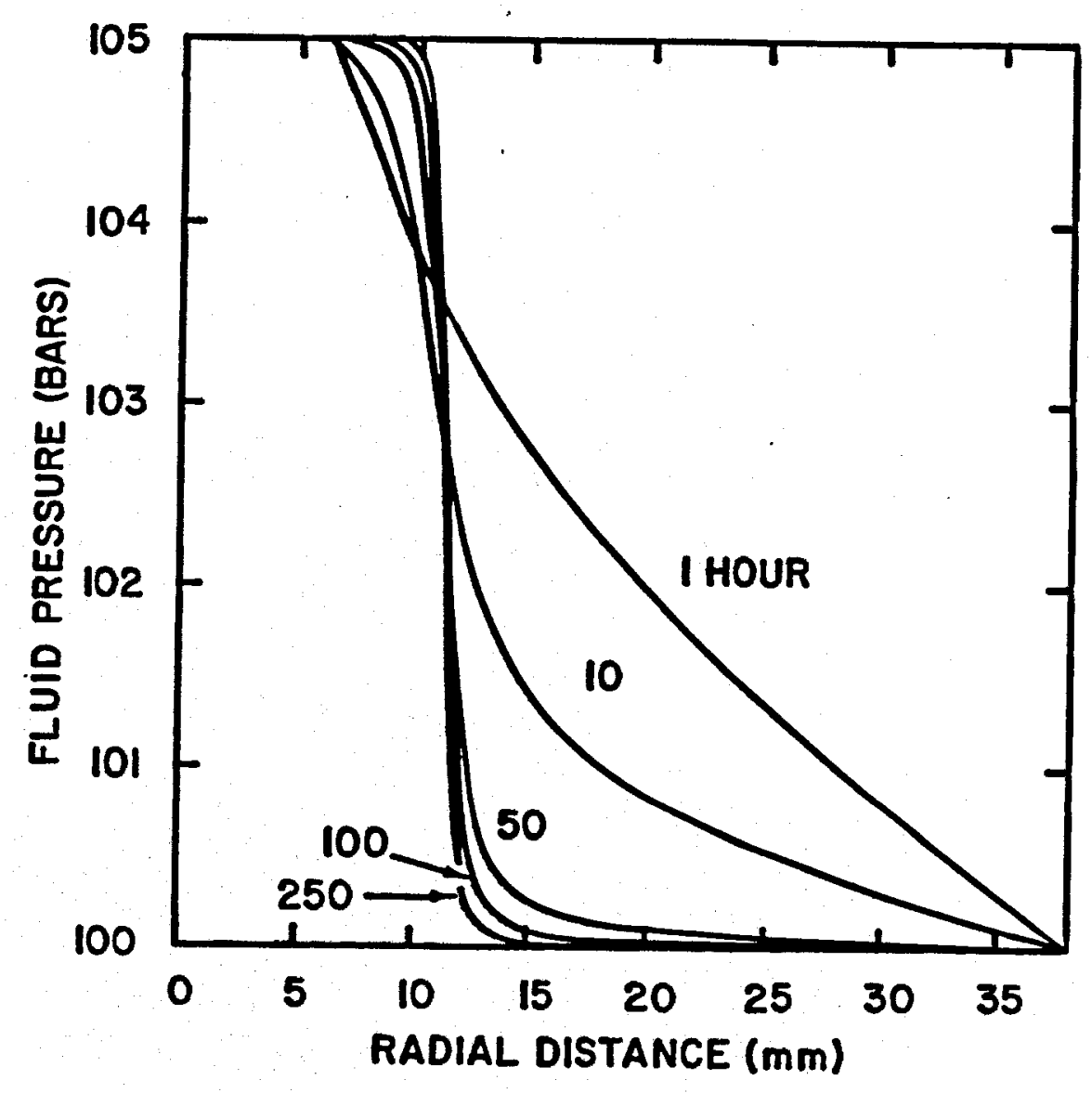

Figure 27 


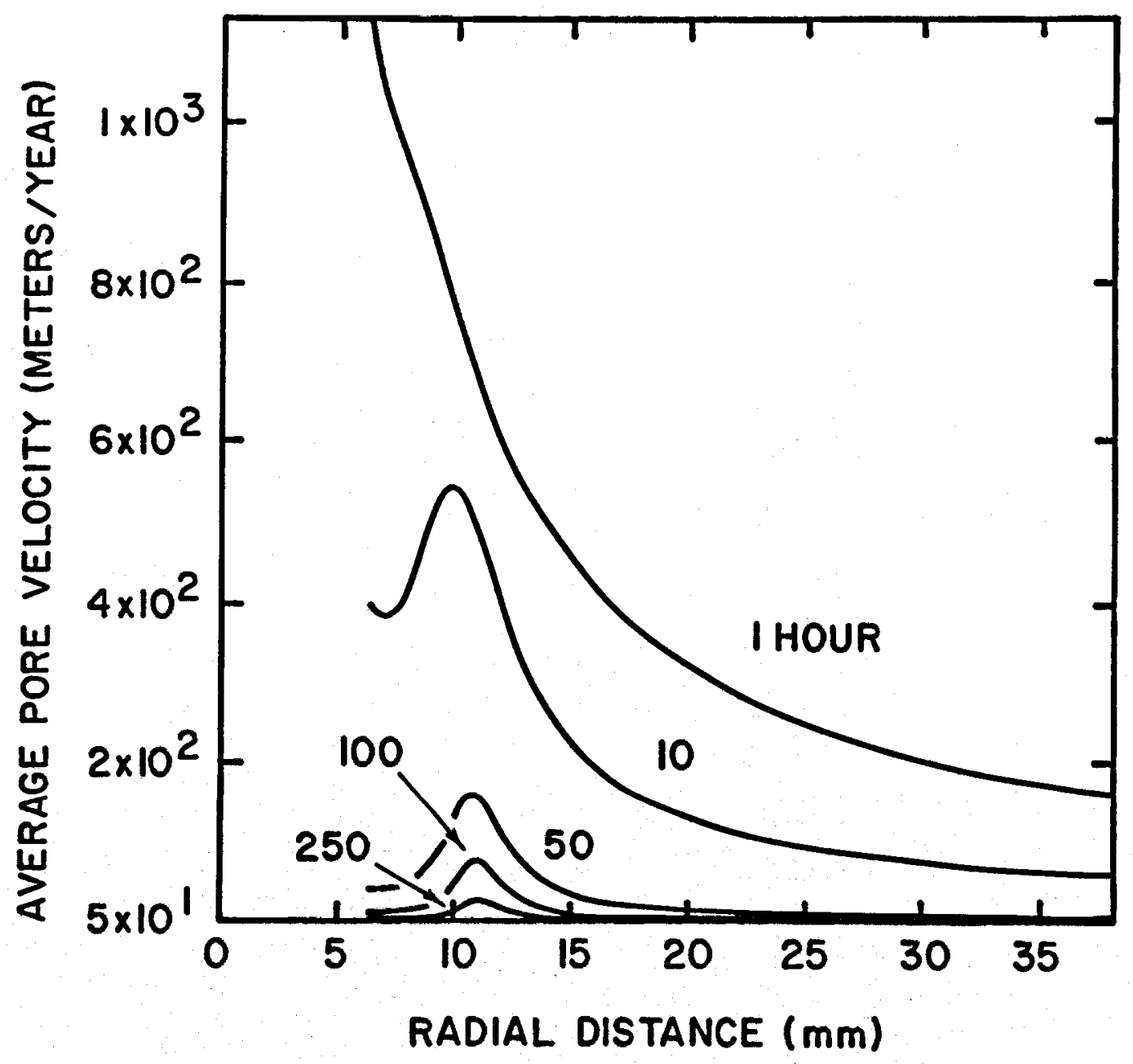

Figure 28 